\title{
GCU
}

Glasgow Caledonian

University

University for the Common Good

\section{Transfer learning networks with skip connections for classification of brain tumors}

Alaraimi, Saleh; Okedu, Kenneth E.; Tianfield, Hugo; Holden, Richard; Uthmani, Omair

Published in:

International Journal of Imaging Systems and Technology

DOI:

10.1002/ima.22546

Publication date:

2021

Document Version

Author accepted manuscript

Link to publication in ResearchOnline

Citation for published version (Harvard):

Alaraimi, S, Okedu, KE, Tianfield, H, Holden, R \& Uthmani, O 2021, 'Transfer learning networks with skip connections for classification of brain tumors', International Journal of Imaging Systems and Technology, vol. 31, no. 3, pp. 1564-1582. https://doi.org/10.1002/ima.22546

\section{General rights}

Copyright and moral rights for the publications made accessible in the public portal are retained by the authors and/or other copyright owners and it is a condition of accessing publications that users recognise and abide by the legal requirements associated with these rights.

Take down policy

If you believe that this document breaches copyright please view our takedown policy at https://edshare.gcu.ac.uk/id/eprint/5179 for details of how to contact us. 


\title{
Transfer Learning Networks with Skip Connections for Classification of Brain Tumors
}

\author{
${ }^{1}$ Saleh Alaraimi, ${ }^{1}$ Kenneth E. Okedu, ${ }^{2}$ Hugo Tianfield, ${ }^{2}$ Richard Holden, ${ }^{2}$ Omair Uthmani \\ ${ }^{1}$ Department of Electrical and Computer Engineering, College of Engineering, National \\ University of Science and Technology, Muscat, Oman \\ ${ }^{2}$ School of Computing, Engineering and Built Environment, \\ Glasgow Caledonian University, Glasgow, UK \\ Email addresses of corresponding authors: salehalaraimi@nu.edu.om or okedukenneth@nu.edu.om
}

\begin{abstract}
This paper presents a transfer learning model via convolutional neural networks (CNN) with skip connection topology, to avoid the vanishing gradient and time complexity, which are usually common in transfer learning networks. Three pre-trained CNN architectures, namely AlexNet, VGG16 and GoogLeNet are employed to equip with skip connections. The transfer learning is implemented through fine-tuning and freezing the CNN architectures with skip connections based on Magnetic Resonance Imaging (MRI) slices of brain tumor dataset. Furthermore, in the pre-processing, a frequency domain information enhancement technique is employed for better image clarity. Performance evaluation is conducted on the transfer learning networks with skip connections to obtain improved accuracy in brain MRI classifications.
\end{abstract}

Keywords: Deep learning, GoogLeNet, AlexNet, VGG, Transfer learning, convolutional neural network $(\mathrm{CNN})$

\section{Introduction}

Brain tumors are the pathological complications caused by uncontrolled cell division, and abnormal growth of tissues. The common types of brain tumor are glioma, meningioma and pituitary. The size of the tumor is quite large in case of glioma, and can lead to short life expectancy [1]. Abnormal growth of brain cell in pituitary gland results in development of pituitary gland tumor that causes abnormal production of pituitary hormones. Due to their intrinsic nature, meningioma, glioma and pituitary gland tumors can occur anywhere in brain tissues. However, these tumors have different shapes, sizes and contrasts [3]. 
Early detection of brain tumor is necessary as delay could lead to death. The detection of location and size of complex cases of brain tumor is a complex task, as the whole treatment plan is based on diagnosis made in the early stages. Thus, locating the exact region of tumor tissues, the extent to which they are spread in nodules, and the grading and type of cancer is necessary. This would help in assessment for the development of treatment plan as well as for the tracking of progression of certain diseases [11-13].

Deep Learning (DL) is considered as one of the most widely used techniques for diagnosis of Alzheimer's, normal and abnormal brain tumor classifications, and stroke lesion segmentation. Thus, nowadays, brain image analysis by radiologists and oncologists often consider DL. In the literature, DL models mostly employ Convolutional Neural Network (CNN) for classification and segmentation of medical images, as it tends to learn the spatial relationship in a hierarchical manner between the pixels [4]. CNN learns how to segment images into many layers; it builds a hierarchy of feature maps by convolving the images through learned filters. . CNN conducts minimal processing and extracts the medical features directly from the pixel images [5- 7].

The effectiveness of DL models in brain tumor imaging and processing is due to the potential to grade and categorize the images [14]. The high performance of CNN enables the radiologist to precisely identify the location, shape, and grade of tumors and assists in the development of effective personalized treatment plan [15].

DL models for neuroimaging involve certain architectures of CNN which are highly efficient by segmenting the medical image into 1,000 images for higher resolution and contrast [16-22]. The CNN models mostly utilized for neuroimaging as well as imaging of biopsy samples for other cancer types include AlexNet, VGG16 and VGG19 [23-27]. DL models are efficient and robust as they precisely address the cancer type, the tissues and neural networks to which tumor has spread, and supplement the data for development of effective treatment plan [28-33].

In [34], a deep learning approach used transfer learning network to classify brain tumors, but skip connection topology was not considered. Reference [35] classified brain tumor images using CNN pre-trained networks. Pre-trained CNN architectures were used as feature extractor for image splicing detection in reference [36]. Although transfer learning networks were able to 
achieve high accuracy in the classifications of brain tumor, the data goes through many layers, which causes vanishing gradient and time complexity.

In this paper, a Transfer Learning (TL) model is presented via convolutional Neural Network $(\mathrm{CNN})$ with skip connection topology, to avoid the vanishing gradient and time complexity, which are common in transfer learning networks.

In particular, three pre-trained CNN architectures, namely GoogLeNet, AlexNet and VGG16 are employed to equip with skip connections.

Also, the $\mathrm{CNN}$ architectures with skip connections have slight changes in the input datasets and in the number of kernels for the output networks. In addition, a frequency domain information enhancement technique is employed for better image clarity in the pre-processing.

Furthermore, transfer learning is implemented through fine-tuning and freezing the CNN architectures with skip connections based on Magnetic Resonance Imaging (MRI) slices of brain tumor dataset Figshare. Performance evaluation is conducted on the fine-tuned CNN architectures with skip connections in brain MRI classifications for common tumors such as meningioma, glioma and pituitary.

\section{Transfer Learning Networks with Skip Connections}

\subsection{Transfer Learning Model via CNN with Skip Connection Topology}

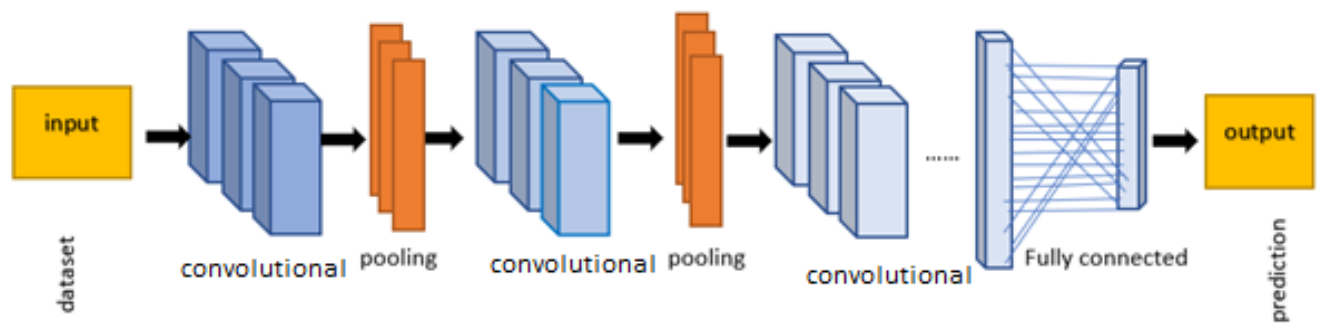

Figure 1. General architecture of CNN 
The CNN architecture, as shown in Figure 1, includes a number of layers having its own functions. In order to train parameters in $\mathrm{CNN}$, the data has to undergo each layer serially. The convolutional layer is known as the first layer in CNN. It uses simple filtering to extract the features of images. The pixels are analyzed based on similarities and relationships that exist in a smaller number of samples.

The main function of pooling layer in $\mathrm{CNN}$ is to decrease the samples required, by keeping the amount of important information, in order to increase the speed of its operations. There are three different types of pooling layers, namely Max, Average and Sum pooling. They play an important role in controlling the overfitting of datasets.

The fully connected layer in $\mathrm{CNN}$ is linking all the activation functions that are present in the previous layers, by bringing appropriate classification. The main task is to retrieve the required information present in the data, i.e., image in this paper. The disadvantage of this layer is the massive requirement of parameters than the previous layers to produce a single output. A fixed number of inputs are sufficient to give a similar output. The aspect ratio input data can be maintained by converting the fully connected layer to convolutional layers of $1 \mathrm{x} 1 \mathrm{kernel}$ and $1 \times 1 \times 1$ in case of 3D images. For better accuracy, the layer uses nonlinear transformation in order to extract features.

The training of deep neural networks is difficult to proceed due to vanishing or exploding gradient issues. Reaching control of deep network by using normalization only may expose degradation problem, while adding a number of layers may increase training errors rate. In 2015, reference [37] introduced a model using Resnet blocks that connects the output of one layer with the input of an earlier layer. This solution is referred to as a skip connection.

In this paper, we employ three pre-trained CNN architectures, namely AlexNet, VGG16 and GoogLeNet based on the Resnet blocks in [37]. In our transfer learning model, the output of each group of layers is connected to the input data with a skip connection, which is a parameter that is also to be learned during the training process. Therefore, in our transfer learning model, the skip connection topology connects input image to groups of layers through a single skip connection in AlexNet and VGG16 networks, and through multiple skip connections in GoogLeNet, at frequent periods. The transfer learning model is implemented with brain tumor dataset for three different classes of brain tumors, i.e., meningioma, glioma and pituitary in this paper. 
Skip connection topology is a nonlinear process that connects neural network layers and skips one or more layers, as shown in Figure 2. The mechanism of skip connections is to skip some layers in neural network and feed the output of one layer, as the input of the next layers. The construction of $F(\mathrm{x})+X$ can be achieved by feed forward neural networks, with "skip connections". The skip connection should learn at least identity mappings from one point in the network, in order to forward them to another point, thus, making the network learn extra $\mathrm{F}(\mathrm{x})$. The process will continue until $\mathrm{F}(\mathrm{x})$ becomes 0 . Consequently, this will help the network to easily learn a mapping closer to 0 than identity mapping.

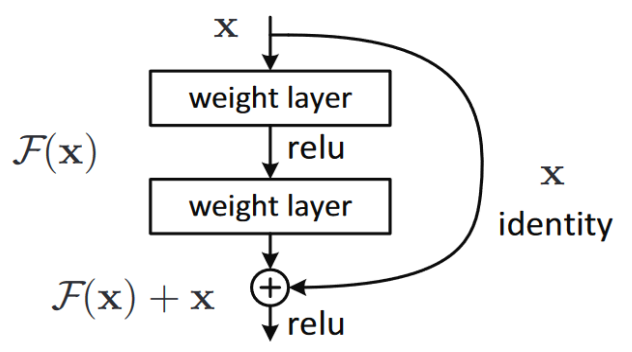

Figure 2. Skip connection topology

In this paper, three pre-trained CNN architectures, i.e., AlexNet, VGG, GoogLeNet, are equipped with skip connections, in order to improve their performances. The CNN with skip or shot connection employed for implementing the transfer learning model is shown in Figure 3. The skip or shot connection works by jumping or transferring data from one convolution layer to another. It should be noted that, when implementing transfer learning, a frequency domain information enhancement technique is employed for better image clarity in the pre-processing for all the CNN architectures with skip connections. In this work, the open source available brain tumor dataset Figshare was used to evaluate the performance of the proposed CNN. The dataset was developed by Cheng in 2017 [38]. 


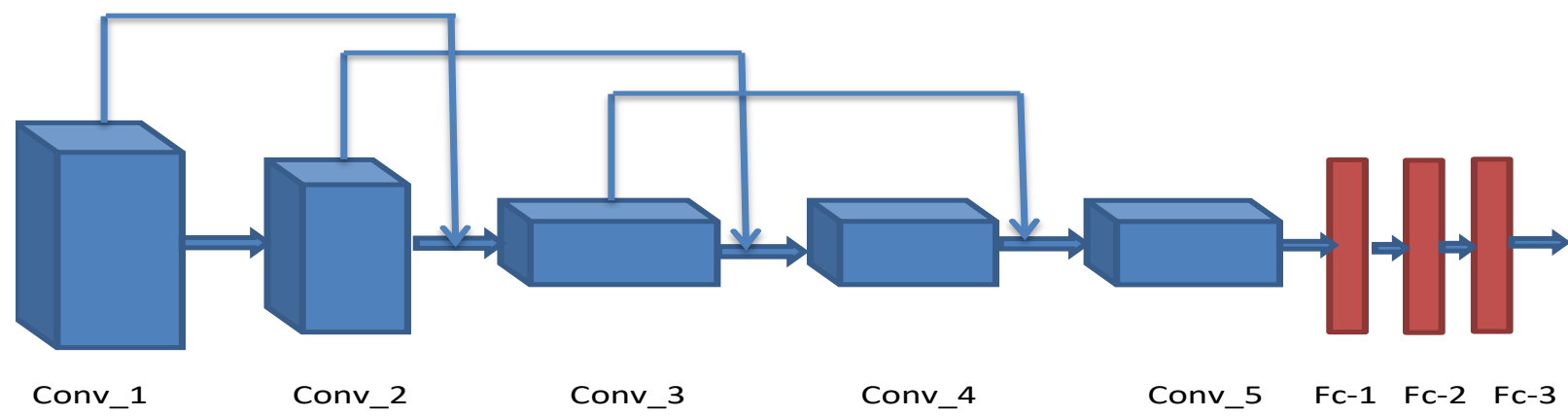

Figure 3. CNN with skip or shot connection for transfer learning

\subsection{CNN Architectures with Skip Connections}

\subsubsection{Alex Net with Skip Connection}

AlexNet is the winner of ISLVRC 2012. It is the first deep neural network that classifies thousands of objects in different classes. The network comprises of convolutional, rectified linear unit, and max-pooling layers. The network consists of five convolutional layers and three fully connected layers. The input size of the image to be fed to this network is $227 \times 227 \times 3$.

Before feeding the input image to AlexNet, images are pre-processed using Z-score image normalization that depends on the mean and standard deviation values of the image pixels. We use the contrast enhancement in addition to the normalization technique to train the images effectively. Contrast enhancement in images refers to the enhancement of the intensity of image pixels, in order to make them more informative. 
In this paper, the contrast enhancement is performed by contrast limited adaptive histogram equalization. Figure 4 shows the architectural model of AlexNet with skip connection, employed in this paper. Generally, the normal AlexNet architecture is pre-trained for 1000 classes. However, the AlexNet with skip connection employed in this paper is changed to 3 classes for cancer detection, based on the dataset classes or requirements for brain tumor.

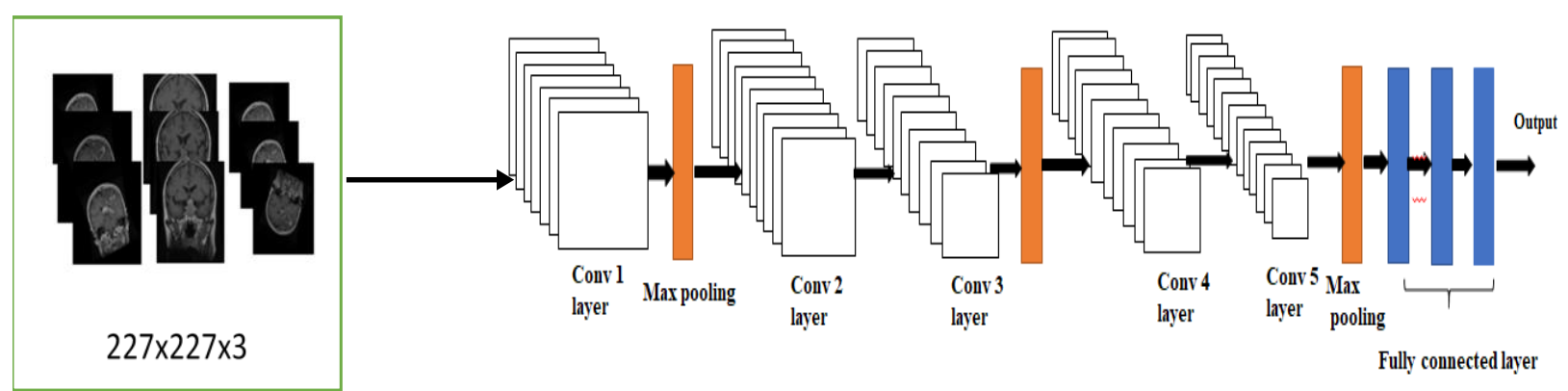

(a) AlexNet architecture

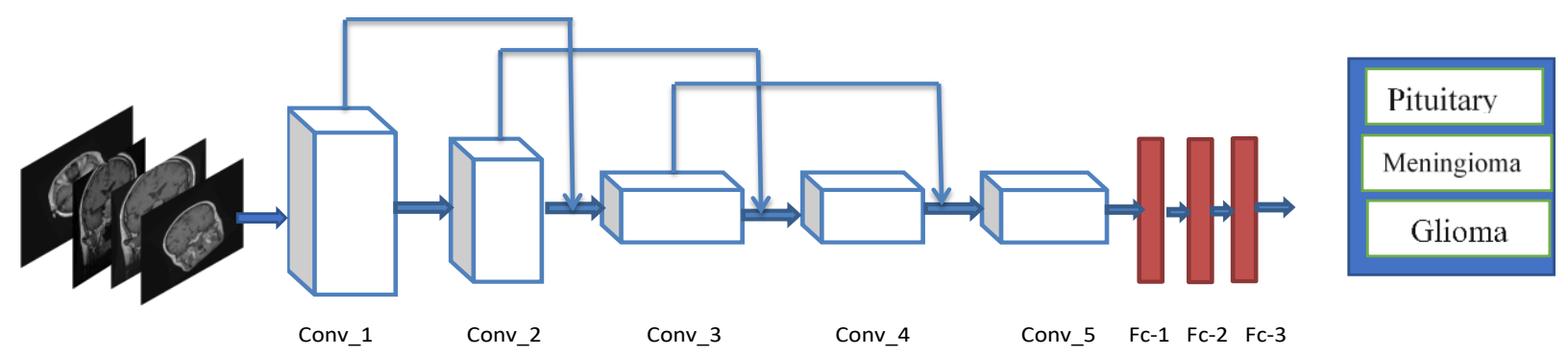

(b) AlexNet with skip connection

Figure 4. Architectural model of AlexNet with skip connection

From Figure 4, the AlexNet with skip connection is different from the normal architecture because of the skip connection, dataset classes, changes in the frequency domain, and the number of kernels used in the network. 
Table 1 lists the summary of the convolutional layers in the AlexNet architecture, and Table 2 shows the number of layers in AlexNet network. The first convolutional layer in the AlexNet architecture has 96 filters with a window size of $11 \times 11$. The second convolutional layer consists of 256 filters with a window size of $5 \times 5$. Likewise, the third, fourth, and fifth layers consist of 384, 384, and 256 filters, respectively. The rectified linear unit (relu) activation function is used between the layers as an activation function that takes the negative values as zero. Therefore, the non-activated neurons become zero which helps to improve the feature extraction for better classification.

For transfer learning, we use the AlexNet which is a pre-trained network, and freeze the layers for fine turning. Thus, the final fully connected layer is set as three as there are three different classes of brain tumors, i.e., Glioma, Meningioma, and Pituitary.

Table 1. Summary of convolutional layers in AlexNet

$\begin{array}{lllll}\text { Convolutional Layer Number } & \text { Number of Filters } & \text { Filter Size } & \text { Stride } & \text { Padding } \\ \text { Convolutional Layer-1 } & 96 & 3 \times 3 & 4 & 0 \\ \text { Convolutional Layer-2 } & 256 & 3 \times 3 & 1 & 2 \\ \text { Convolutional Layer-3 } & 384 & 3 \times 3 & 1 & 1 \\ \text { Convolutional Layer-4 } & 384 & 3 \times 3 & 1 & 1 \\ \text { Convolutional Layer-5 } & 256 & 3 \times 3 & 1 & 1\end{array}$

Table 2. Summary of layers in AlexNet

$\begin{array}{llll}\text { Layer type } & \text { Feature Map } & \text { Kernel Size }+ \text { stride } & \text { Activation } \\ \text { Input Image } & 1 & - & - \\ \text { Convolution } & 96 & 3^{*} 3-\text { stride } 4 & \text { relu } \\ \text { Max Pooling } & 60 & 3^{*} 3-\text { stride 2 } & \text { relu } \\ \text { Convolution } & 256 & 3^{*} 3-\text { stride 1 } & \text { relu } \\ \text { Max Pooling } & 256 & 3^{*} 3-\text { stride 2 } & \text { relu } \\ \text { Convolution } & 384 & 3^{*} 3-\text { stride 1 } & \text { relu } \\ \text { Convolution } & 256 & 3^{*} 3-\text { stride 1 } & \text { relu } \\ \text { Max Pooling } & 256 & 3^{*} 3-\text { stride 2 } & \text { relu } \\ \text { FC } & - & - & \text { relu } \\ \text { Softmax } & - & - & \text { Softmax }\end{array}$

\subsubsection{VGG16 with Skip Connection}


VGG16 is a sixteen-layer CNN architecture. Similar to AlexNet, it is made up of convolutional, max-pooling Relu layers [39]. The number of filters changes from 64 to 512, and there are three fully connected layers. All the convolutional layers have a filter window size of $3 \times 3$. VGG16 has fully connected layers, the layer parameters and the number of layers is higher compared to AlexNet. In the VGG16 architecture, the first, second convolutional layers have the same filter size of 64 , the third and fourth convolutional layers have 128, the fifth, sixth and seventh convolutional layers have 256 filters. Likewise, the eighth, ninth, tenth, eleventh, twelfth and thirteenth layers have 512 filters. At the end, it consists of three fully connected layers.

The computational complexity of this VGG16 architecture is higher compared to other networks, since it consists of two stages 512 filters each. To perform fine-tuning for transfer learning, the final fully connected layer is tuned to produce three output classes instead of 1000 classes. The activation function used here is the rectified linear unit. Each convolutional layer extracts suitable features and it is a pyramid architecture that helps to improve the classification accuracy.

Figure 5 shows the VGG16 with skip connection topology employed in this paper, with an input dataset of 224 x 224, and kernel output for the datasets under consideration. Table 3 shows the number of layers in VGG16 network.

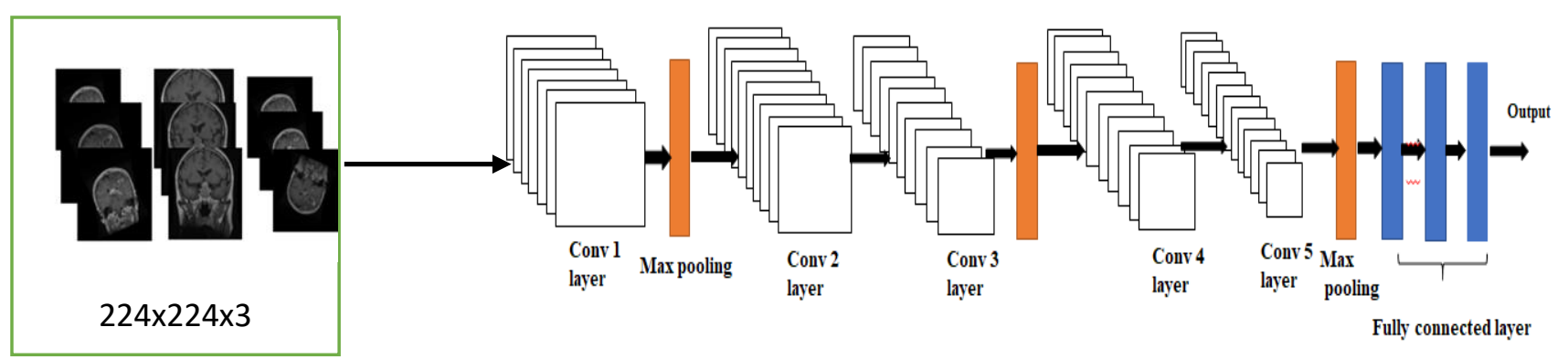

(a) VGG16 architecture 


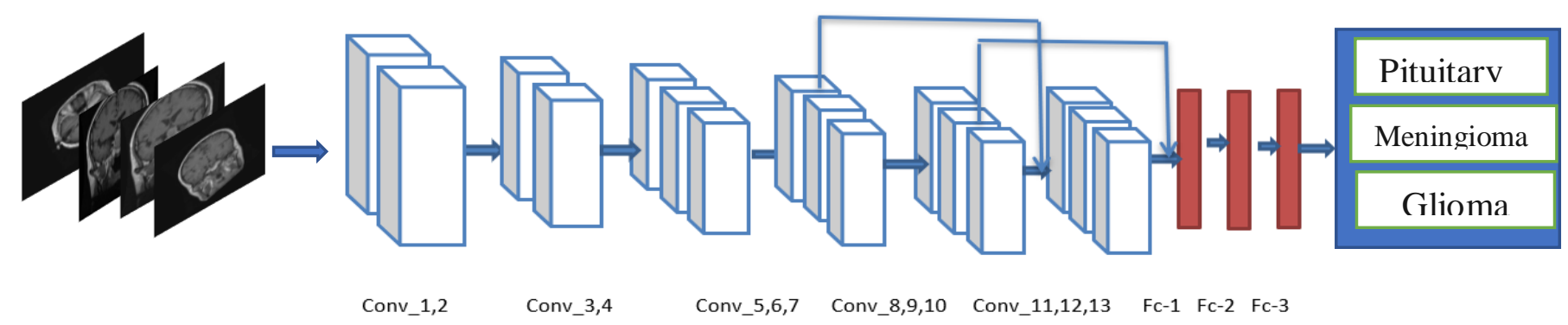

(b) VGG16 with skip connection

Figure 5. Architectural model of VGG16 with skip connection

Table 3. Summary of layers in VGG16

\begin{tabular}{|c|c|c|c|}
\hline Layer type & Feature Map & Kernel Size + stride & Activation \\
\hline Input Image & 1 & - & - \\
\hline Convolution & 64 & $3 * 3$ - stride 4 & relu \\
\hline Convolution & 64 & $3 * 3$ - stride 4 & relu \\
\hline Max Pooling & 64 & $3 * 3-$ stride 2 & relu \\
\hline Convolution & 128 & $3 * 3$ - stride 4 & relu \\
\hline Convolution & 128 & $3 * 3$ - stride 4 & relu \\
\hline Max Pooling & 256 & $3 * 3-$ stride 2 & relu \\
\hline Convolution & 256 & $3 * 3$ - stride 1 & relu \\
\hline Convolution & 256 & $3 * 3$ - stride 1 & relu \\
\hline Convolution & 256 & $3 * 3$ - stride 1 & relu \\
\hline Max Pooling & 256 & $3 * 3$ - stride 2 & relu \\
\hline Convolution & 512 & $3 * 3$ - stride 1 & relu \\
\hline Convolution & 512 & $3 * 3$ - stride 1 & relu \\
\hline Convolution & 512 & $3 * 3-$ stride 2 & relu \\
\hline Max Pooling & 512 & $3 * 3$ - stride 1 & relu \\
\hline Convolution & 512 & $3 * 3$ - stride 1 & relu \\
\hline Convolution & 512 & $3 * 3-$ stride 2 & relu \\
\hline Convolution & 512 & & \\
\hline Max Pooling & 256 & $3 * 3$ - stride 2 & relu \\
\hline $\mathrm{FC}$ & - & - & relu \\
\hline Softmax & - & - & Softmax \\
\hline
\end{tabular}

\subsubsection{GoogLeNet with Skip Connection}


GoogLeNet with a shot connection is a network architecture in which the previous layer connections will be added with the current layers to avoid the vanishing gradients. Here, the previous layer features are added with the present layer features such that there is no feature loss. Also, the present layer feature values will improve the learning of effective features.

GoogLeNet [3, 40] has nine inception modules. Furthermore, each inception module contains one max-pooling layer and six convolutional layers. From this, four convolutional layers are used for dimension reduction. Relu activation function is applied in all the fully connected layers and dropout regularization is used in the fully connected layers.

GoogLeNet has 6.8 million parameters, because of additional feature learning. It comprises of nine inception modules, two convolutional layers, four max-pooling layers, one convolutional layer for dimension reduction, one average pooling, two normalization layers, one fully connected layer, and finally a linear layer with softmax activation in the output.

The performance of GoogLeNet is more accurate than other architectures, i.e., VGG and AlexNet. Moreover, it is more precise than AlexNet on the original ILSVRC dataset.

The GoogLeNet with skip connection employed in this paper is given in Figure 6. Table 4 lists the summary of layers in the GoogLeNet. 


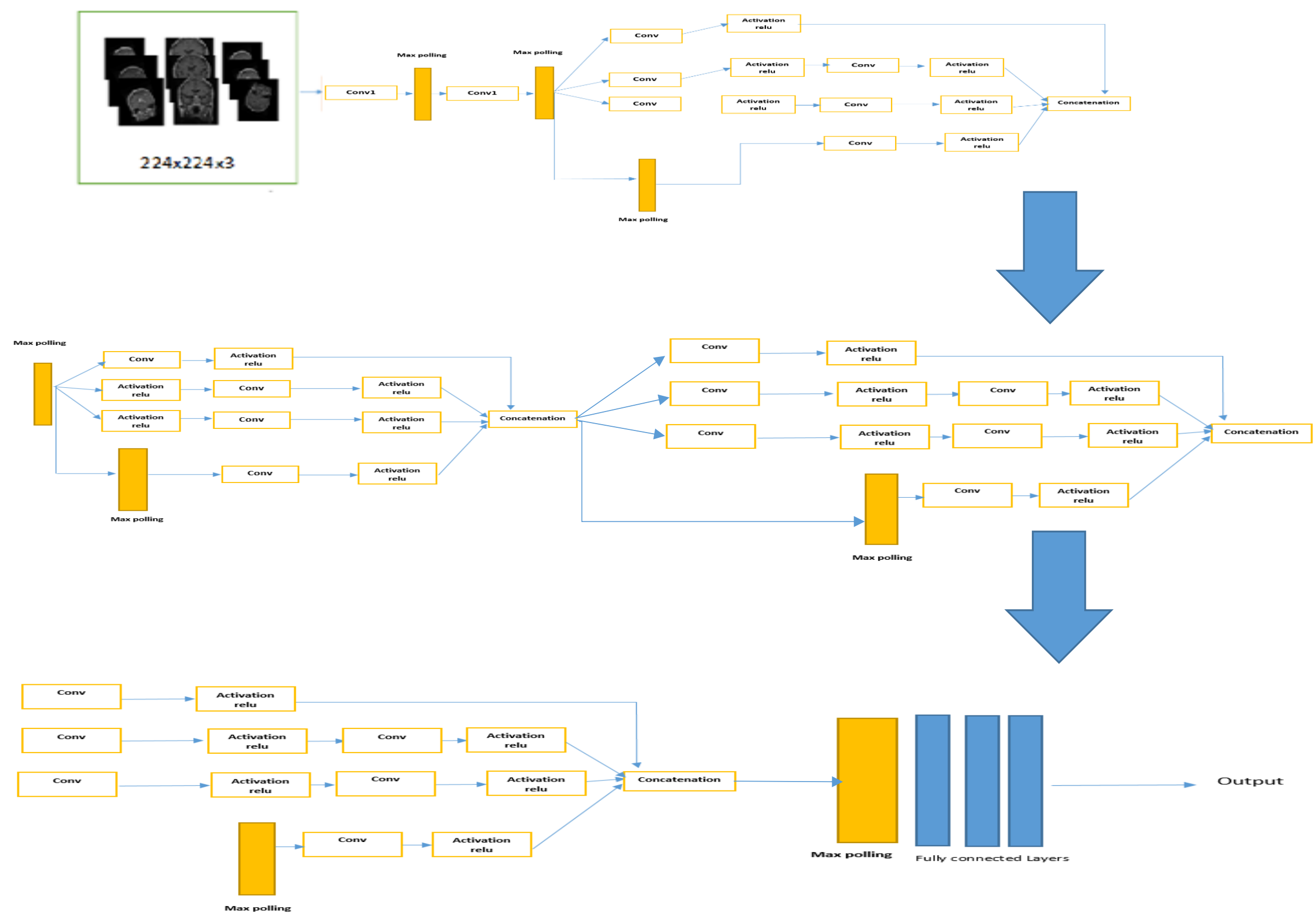

(a) GoogLeNet architecture 


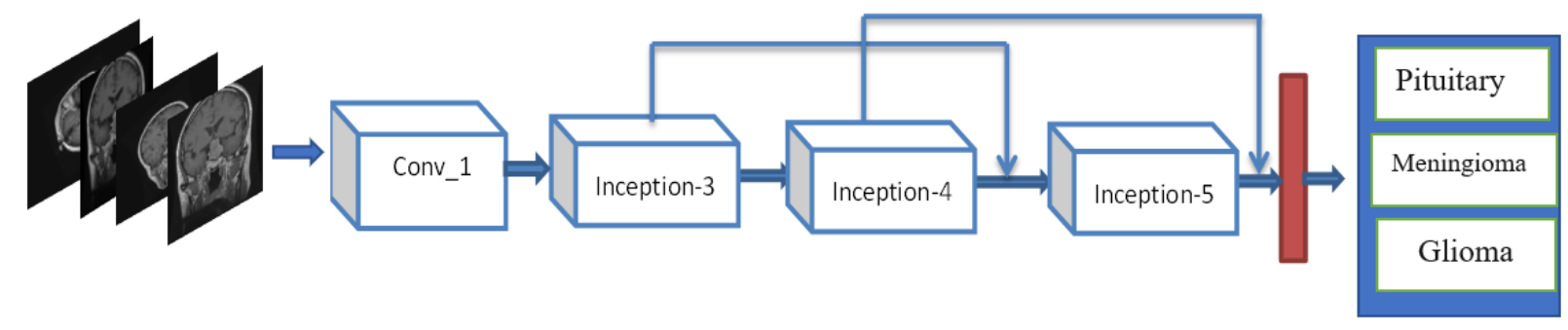

(b) GoogLeNet with skip connection

Figure 6. Architectural model of GoogLeNet with skip connection

Table 4. Summary of layers in GoogLeNet

\begin{tabular}{|c|c|c|c|}
\hline Layer type & Feature Map & Kernel Size + stride & Activation \\
\hline Input Image & 1 & - & - \\
\hline Convolution & 64 & $3 * 3$ - stride 4 & relu \\
\hline Max pooling & 64 & $3 * 3$ - stride 4 & relu \\
\hline Convolution & 60 & $3 * 3-$ stride 2 & relu \\
\hline Max Pooling & 128 & $3 * 3$ - stride 4 & relu \\
\hline Inception (3a) & 128 & $3 * 3$ - stride 4 & relu \\
\hline Inception (3b) & 256 & $3 * 3$ - stride 2 & relu \\
\hline Max Pooling & 256 & $3 * 3$ - stride 1 & relu \\
\hline Inception (4a) & 256 & $3 * 3$ - stride 1 & relu \\
\hline Inception (4b) & 256 & $3 * 3$ - stride 1 & relu \\
\hline Inception (4c) & 256 & $3 * 3$ - stride 2 & relu \\
\hline Inception (4d) & 512 & $3 * 3$ - stride 1 & relu \\
\hline Inception (4e) & 512 & $3 * 3-$ stride 1 & relu \\
\hline Max Pooling & 512 & $3 * 3$ - stride 1 & relu \\
\hline Inception (5a) & 512 & $3 * 3-$ stride 1 & relu \\
\hline Inception (5b) & 512 & $3 * 3-$ stride 2 & relu \\
\hline Avg Pooling & 256 & $3 * 3$ - stride 2 & relu \\
\hline FC & - & - & relu \\
\hline Softmax & - & - & Softmax \\
\hline
\end{tabular}




\subsection{Training Strategies of CNNs with Skip Connections}

In this section, the training methods used in the transfer learning networks are discussed. To increase the classification performance, all the three pre-trained networks are fine-tuned for transfer learning. In all the pre-trained networks, the fully connected layers are fixed as three. Learning rate and other learning parameters are also changed to achieve better results. In all three pre-trained networks, activation has been analyzed, but the GoogLeNet activation function is more visible than the other two networks. Three different patch sizes, namely 16, 32, and 64 are analyzed, and it is finally fixed with 32 in this paper.

The Adam optimization algorithm is used here for training. It helps to reduce the gradient value as quickly as possible. It is the combination of two optimization algorithms, such as gradient descent with momentum and RMSprop. This combined optimizer effectively works for the fine tuning of hyper parameters. Also, its low memory requirements help to improve the training accuracy with fewer epochs.

The loss function is an important criterion in training to recognize the class labels. This loss function needs to be minimized. The loss function used in this paper is cross-entropy loss function [41], which is defined as

$\mathrm{L}(\mathrm{w})=\sum_{i=1}^{N} \sum_{c=1}^{4}\left\{-y_{i c} \log f_{c}\left(x_{i}\right)\right\}+\in\|w\|_{2}^{2}$

$\mathrm{f}_{\mathrm{c}}\left(\mathrm{x}_{\mathrm{i}}\right)$ - predicted probability of class $\mathrm{c}$ for image $\mathrm{x}$

The training algorithm is given as Algorithm 1.

Algorithm 1. Training algorithm of CNN with skip connection for transfer learning

1. Input: Input data samples for Training Datasets $(T D s)$ with the number of images $n I m$, class labels $\mathrm{Cl}$ (glioma, meningioma, and pituitary), validation datasets (VDs), learning rate $R a$,

2. Output: Feature map, performance metrics such as accuracy, precision, recall, etc.

3. Reduce the cross entropy, train the network with Adam optimizer.

4. Loss is computed on the training datasets $T D s$ and the validation datasets $V D s$ by backpropagation algorithm based on the class labels.

5. Initialize the learning rate $R a$ 
6. For $z=1$ to the number of images $n I m$

a. Take the feature map for the final convolutional layer

b. Find softmax values of the output layer

7. Return Loss Lr, Accuracy Ac, feature map

The performance of the deep network can be improved by increasing the size of the training data. Image augmentation can be carried out to increase the size of the training data. If there is not much training data, image augmentation can be obtained by resizing, rotating, and flipping operations. However, while we increase the training data, the data memory requirement on the computer and the computation time will be high.

The selection of patch size is very important to get the optimal performance of the network. In this brain tumor detection problem, we set the minimum patch size as 32, which will help to improve the detection rate. In some scenarios, increasing the patch size leads to a high misclassification rate because most of the feature maps will be redundant, which decreases the optimal performance of the network.

Generally, it is not enough that the entire datasets are given to the network for only one time, because the network needs to calculate the loss for different training samples. Therefore, the maximum epoch must be higher than one. The number of epochs increases the weight value of the network. The epoch value should be increased until you get the training accuracy higher or 100. Here we set the maximum epoch as 200.

For the pre-processing, two different methods are used here. Both are in the frequency domain. The two methods are histogram equalization and normalization. Stationary Wavelet Transformation (SWT) is used for frequency-domain conversion. These two methods are carried out in the frequency domain only. Four sub-bands are obtained in the frequency domain. Initially, the input image is converted from the spatial domain to the frequency domain by wavelet transformation, then the equalization is carried out on all the sub-bands and inverted to the original domain. After the equalization, the same frequency domain process is carried out for $\mathrm{Z}$ score normalization in all the sub-bands.

Figure 7 shows frequency domain information enhancement on the image improvement in the pre-processing, , which gives higher resolution to fit into the training process, while Figure 8 is the frequency information enhancement process. 


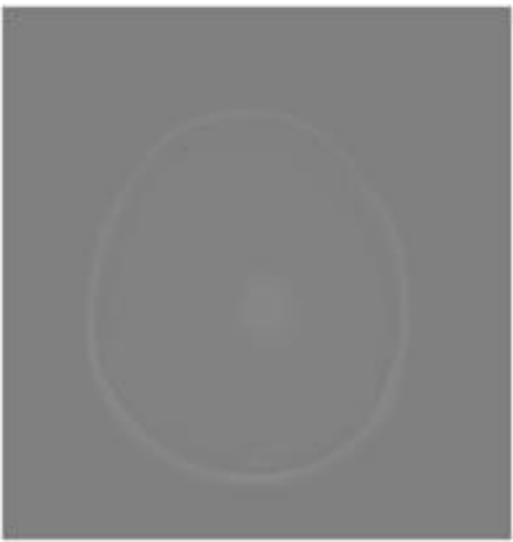

(a) Original image

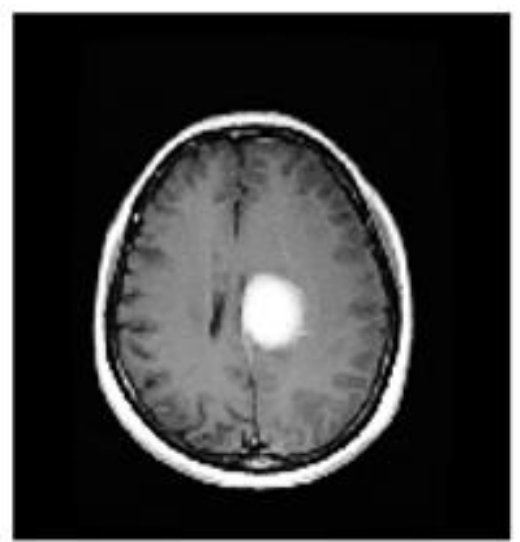

(b) Contrast enhanced image

Figure 7. Result of contrast enhancement

Equalization and normalization give contrast-enhanced images for better training. The $\mathrm{Z}$ score normalization is defined by $[42,43]$

$$
Z s c=\frac{X-\text { mean }}{s t d}
$$

$$
\text { Zsc-Normalized outcome ,X-input data }
$$

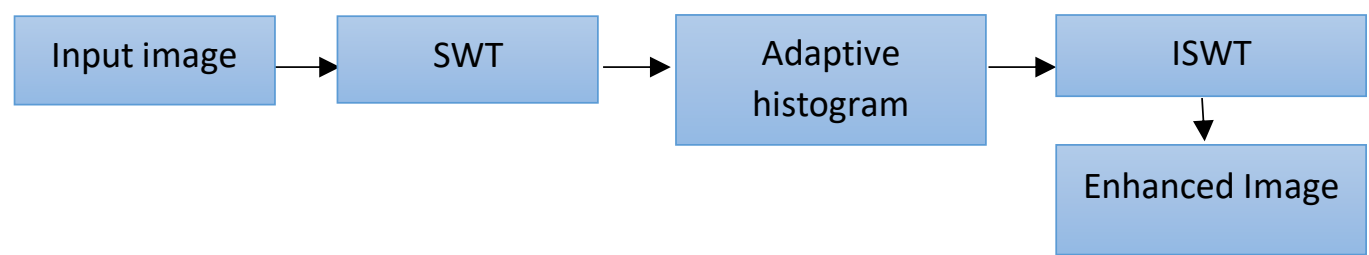

Figure 8. Frequency domain information enhancement process in the pre-processing SWT - Stationary Wavelet Transformation, ISWT - Inverse Stationary Wavelet Transformation 
Once the image data augmentation is completed, the network is fed with this huge amount of training data. This increases the amount of training data and leads to improve classification performance.

The convolution layers are the second layer of the network after the input layer, which helps to extract special features such as low-level, medium, and high-level features. Each convolutional layer extracts different levels of features which helps to train the network in a better way. The three pre-trained CNN architectures, AlexNet, VGG16, and GoogLeNet, use the pre-trained weights that are transferred from one layer to another layer. For fine-tuning, the final fully connected layer of the three pre-trained networks is replaced by 3 classes instead of 1000 classes. The pre-trained network weights are transferred to the target dataset and there is no need for retraining the network. The significance of transfer learning is that a smaller number of datasets are needed under this circumstance because the weights are transferred. In this paper, the softmax is used as the classification layer.

Image data augmentation is needed for improving the training accuracy, by increasing the number of training images. In this paper, we have used five different types of image processing techniques which are image rotation, cropping, flipping, scaling, and translation. In total there are 100 gliomas, 120 meningioma, and 150 pituitaries. Preprocessing is carried out on these three classes and augmented to get a total of 1500 images. Figure 9 shows the image data augmentation. In Figure 9(a), the data is rotated 10 degrees, while Figure 9(b) is normal orientation, Figure 9(c), is flipped from upper orientation to laser and Figure 9(d) is rotated by 45 degrees. 


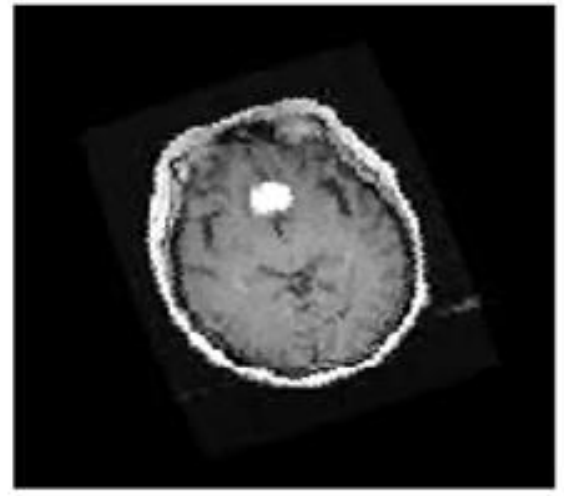

(a) Rotation of 10 degrees

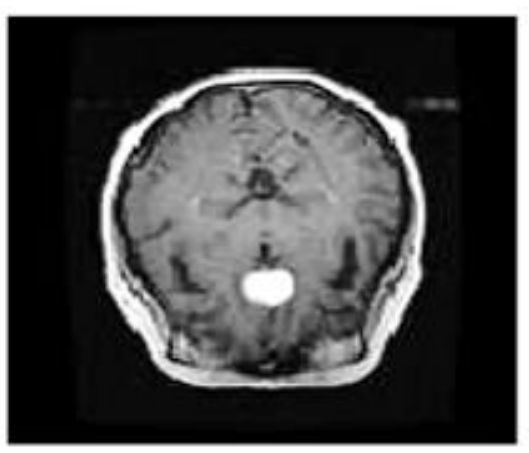

(c) Flip Rotation

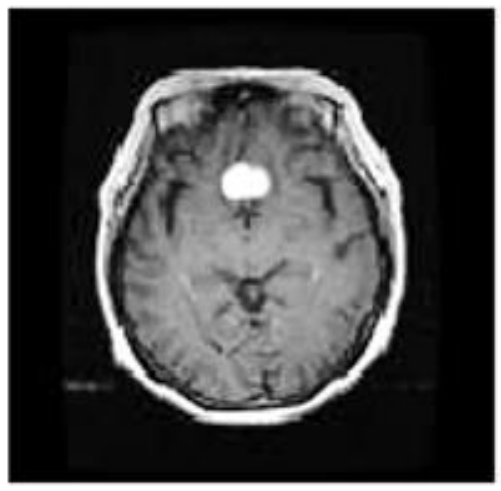

(b) Normal Rotation

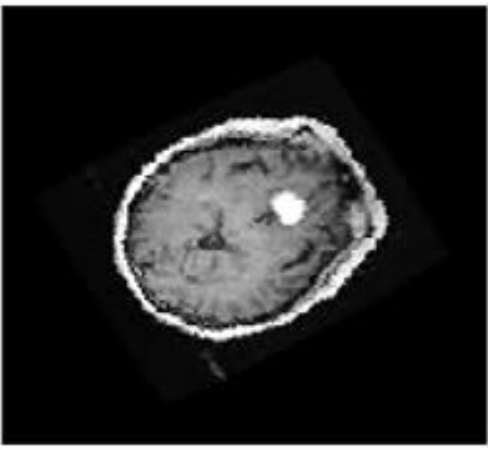

(d) Rotation of 45 degrees

Figure 9. Augmented image data in different forms

\section{Performance Evaluation}

In order to implement our transfer learning model, Matlab 2020a is employed. The Matlab tool is used with single graphical processing unit having multiple processers of $2.6 \mathrm{GHz}$ and 16GB RAM, 1TB of HDD. This section presents a set of the experiments that are carried out using brain tumor dataset Figshare for brain tumor classification and detection. We evaluate the performance of three pre-trained CNN architectures, AlexNet, VGG16 and GoogLeNet, using augmented image slices of brain tumor dataset. These pre-trained CNN architectures are used to 
deploy the transfer learningmodel, in order to extract the rich features. Then, the confusion matrices are discussed for evaluation performance of the CNN architectures with skip connections.

In the experiments, we use the open-source brain tumor dataset Figshare to evaluate the performance of the $\mathrm{CNN}$ architectures with skip connections. The dataset was developed by Cheng in 2017. The number of brain MRI is 3064 collected from 233 patients, with three different types of brain tumors, i.e., meningioma, glioma and pituitary. The images are distributed as follows: 930 images for pituitary, 708 images for meningioma and 1426 images for glioma. The main format is ".mat", each file including structure with a patient ID, unique label that denotes the type of brain tumor, $512 \times 512$ image data in unit 16 format, a vector containing a tumor boundary, with discrete dot coordinates, and a ground truth in a binary mask image.

MRI is an imaging technique employed in medical sciences to analyze both anatomy and physiological processes of some parts or the entire human body. The scanners associated with this kind of imaging technique have sophisticated radio waves, magnetic fields and gradients, which enable capturing of images of human organs.

The CNN architecture takes MRI images as an input unit in our experiments; hence, we only use the image data from the ".mat" files after extraction and resizing, to fit the model requirements. The CNN architecture performs automatic feature extraction using convolutional layers without human intervention. CNN generally performs better in comparison to the classic image processing methods.

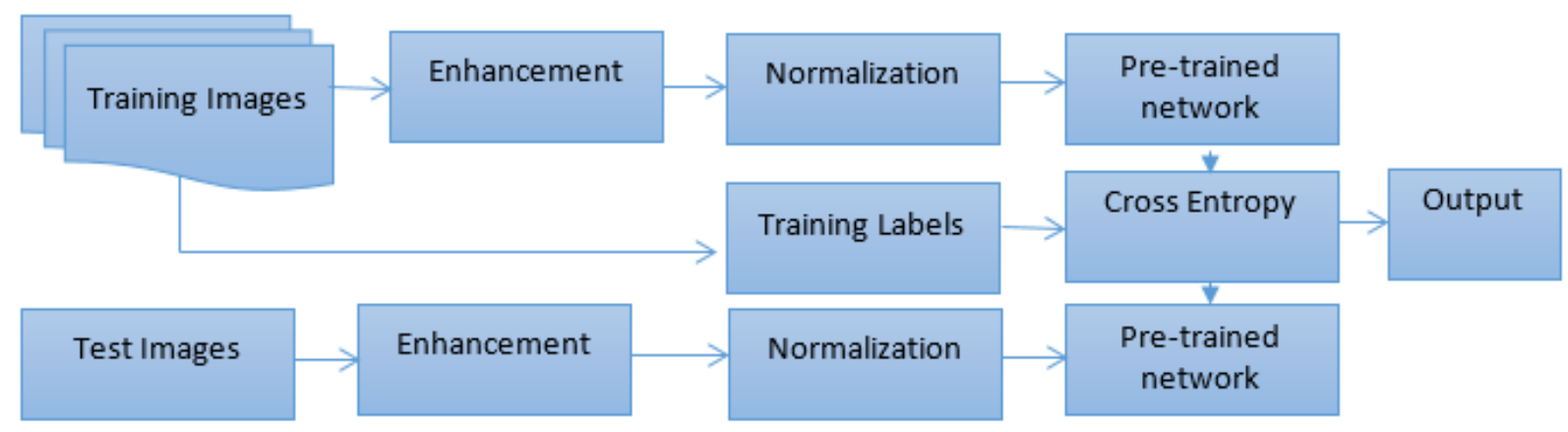


Figure 10. Training and testing processes in experimental setup

The experimental setup shown in Figure 10 contains both the training and the testing processes. The enhancement and normalization are carried out in both the training and the testing. Then, feature extraction is carried out using the pre-trained network, namely AlexNet, VGG16, GoogLeNet architectures. Finally, the cross-entropy loss function is computed to differentiate the input classes with the help of training data labels. This process is repeated for testing images as well, to identify the classes, whether it is glioma, meningioma, or pituitary.

Various experiments are conducted to evaluate the performance of the system. In the first experimental scenario, the images are randomly split as $70 \%$ for training and $30 \%$ for testing. In the other experimental scenario, $80 \%$ of the images are used for training and $20 \%$ for testing. Both experimental scenarios provide promising results, but the second experimental scenario provides results which are slightly better than the first experimental scenario.

In this paper, we also evaluate different optimizers, namely Stochastic Gradient Decent with Momentum (SGDM), Adam, and RMSprops. GoogLeNet using Adam optimizer provides better results compared to other networks. The experiments are conducted on both original and augmented data independently. After freezing some of the layers in all three pre-trained networks independently, the impact of the remaining layers is also analyzed.

The AlexNet, VGG16, and GoogLNet are experimentally evaluated based on the performance metrics such as accuracy, sensitivity, specificity and F-score. The performance metrics are stated below.

True positive $(\mathrm{Tp})=$ Number of identified positive samples that are truly positive

False positive $(\mathrm{Fp})=$ Number of identified positive samples that actually are negative

True negative $(\mathrm{Tn})=$ Number of identified negative samples that are truly negative

False negative $(\mathrm{Fn})=$ Number of identified negative samples that actually are positive

Accuracy is the ability to discriminate the different classes correctly. To estimate the accuracy of a network, the proportion of true positive and true negative among all samples is computed, that is, 


$$
\text { Accuracy }=(T p+T n) /(T p+T n+F p+F n)
$$

Sensitivity is the ability to see the unique independent class samples properly. To estimate it, we should compute the proportion of true positive in unique independent samples, that is,

$$
\text { Sensitivity }=T p /(T p+F n)
$$

Specificity is the ability to identify the negative samples properly. To estimate it, we must compute the proportion of true negative in normal cases, that is,

$$
\text { Specificity }=T n /(T n+F p)
$$

\subsection{Performance of AlexNet with Skip Connection}

We have taken individual alternate layers from AlexNet and obtained the performance metrics as presented in Table 5. From Table 5, we can see that the increment of convolutional layers leads to accuracy improvements, that is, Conv_1 gives $50 \%$ accuracy but conv_3, conv_4 give $63.5 \%$ and $95 \%$, respectively, which are better than conv_1; so the features learned from the conv_5 layer are better than all other layers. The overlapping feature maps are eliminated by the dropout layers, so it provides better performance.

Table 5. Performance of AlexNet with skip connection

$\begin{array}{lcrccc}\text { Layers } & \text { Accuracy (\%) } & \begin{array}{l}\text { Sensitivity } \\ (\%)\end{array} & \begin{array}{l}\text { Specificity } \\ \text { (\%) }\end{array} & \text { F-score (\%) } & \text { Precision (\%) } \\ \text { Conv_1 } & 50 & 55 & 78 & 59 & 56 \\ \text { Conv_3 } & 63.5 & 70.2 & 84 & 72.5 & 72 \\ \text { Conv_5 } & 95 & 94.2 & 98 & 93 & 94 \\ \text { FC_6 } & 96.1 & 95.2 & 98.2 & 94.5 & 95 \\ \text { FC_7 } & 92 & 91 & 96 & 92.2 & 91\end{array}$

The performances of the intermediate layers are analyzed after training the pre-trained AlexNet architecture (trained on million images) on the brain tumor dataset. It is carried out by extracting and utilizing the features learnt from the individual intermediate layers for brain tumor classification. The features learnt from the intermediate convolutional layers such as conv_1, conv $\_3$, conv 5 and two fully connected layers are separately used, by freezing the remaining layers for brain tumor classification, using the machine learning classifier. Here, the Support Vector Machine (SVM) classifier is used to extract the image features. It is understood that 
taking features from conv_3 layer includes the mid-level features learnt from the lower levels after performing successive convolution, i.e., conv_1 to conv_3.

From Table 5, after freezing some of the layers in the AlexNet, the performance of layers such as, conv_1 to conv_3, conv_1 to conv_ 5, conv_1 to FC_6 and conv_1 to FC_7 is analyzed. It is observed that, when the number of convolutions is increased, the results of the fully connected layer FC_6 have higher performance metrics, i.e., accuracy of 96.1\%, sensitivity of $95.2 \%$, and specificity of $98.2 \%$. Also, it has a higher precision of $95 \%$ and an F-score of $94.5 \%$. In conv_1, the network extracts low-level features such as edges, textures, and at the output of conv_3, certain mid-level features are extracted. Finally, at the end of conv_5, high-level features such as the entire region of interest are extracted. Therefore, the first FC_6 layer is able to produce better performance after the extraction of the features from the lower level to a higher level.
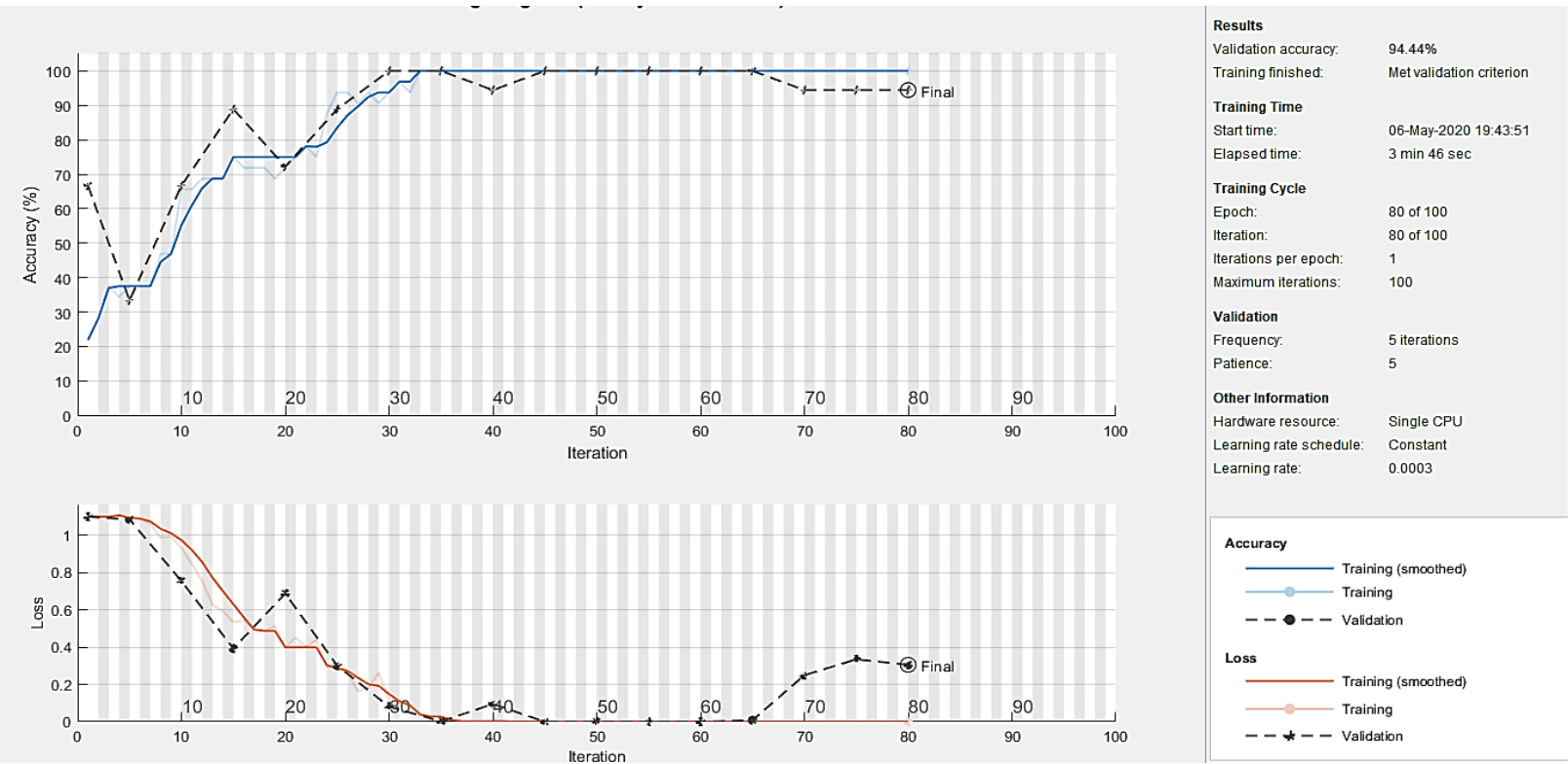

Figure 11. Training progress of AlexNet with skip connection

Figure 11 shows the training progress in which the training accuracy is getting $100 \%$ when the number of epochs is 200 and the loss is reducing up to 0.003 . Here, the learning rate is 
0.0003 and the patch size is 32 . Validation accuracy is also progressing in a promising manner. There is no quick loss variation.

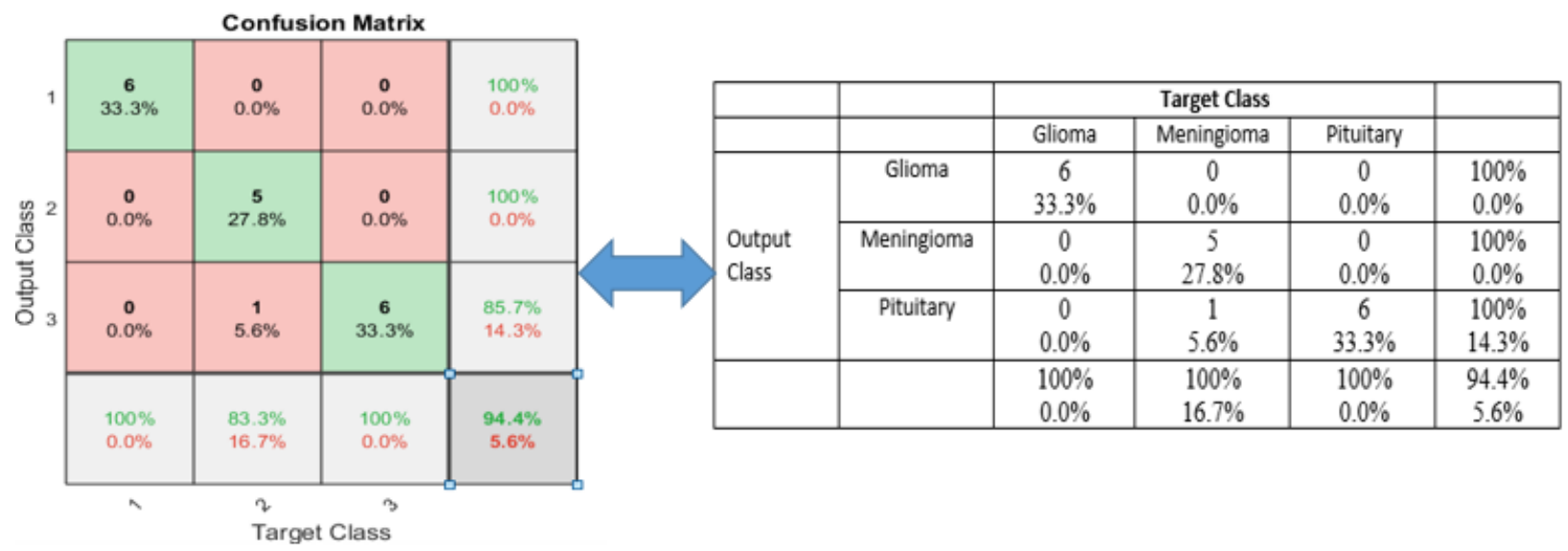

Figure 12. Confusion matrix for AlexNet with skip connection

The numerical computation in the confusion matrix are obtained as follows:

True Positive $(\mathrm{Tp})=6$; True Negative $(\mathrm{Tn})=5+0+1+6=12, \quad$ False Positive $(\mathrm{Fp})=0$ and False Negative $(F n)=0$.

The True Positive $=\mathrm{Tp} /$ sample total; $6 / 18=33.33 \%$. In our case the number of trained samples has been 18 Glioma out of 20 and 17 out of Meningioma; $5 / 17=27.8 \%$ and 6 from Pituitary $6 / 18=33.33 \%$.

From Figure 12, the digits 1, 2 and 3 represent the different classes of brain tumors, namely Glioma, Meningioma and Pituitary. The performance of this brain tumor classification system is visualized by the confusion matrix as shown in Figure 12. Here, the brain tumor is classified into three classes. Therefore, the confusion matrix has a $3 \times 3$ matrix. From Figure 12, in the experiment, among the 60 test samples, 6 Glioma test samples are identified as Glioma, 3 samples are identified as Meningioma, and 4 as Pituitary. Likewise, for Meningioma classes: 5 samples are accurately identified as Meningioma, 2 samples as Glioma, and 2 as Pituitary. For Pituitary class: 6 samples are identified as Pituitary, 2 samples as Glioma, and 3 samples are identified as Meningioma, respectively. So, the overall accuracy for the fine-tuned AlexNet is $94.4 \%$. 


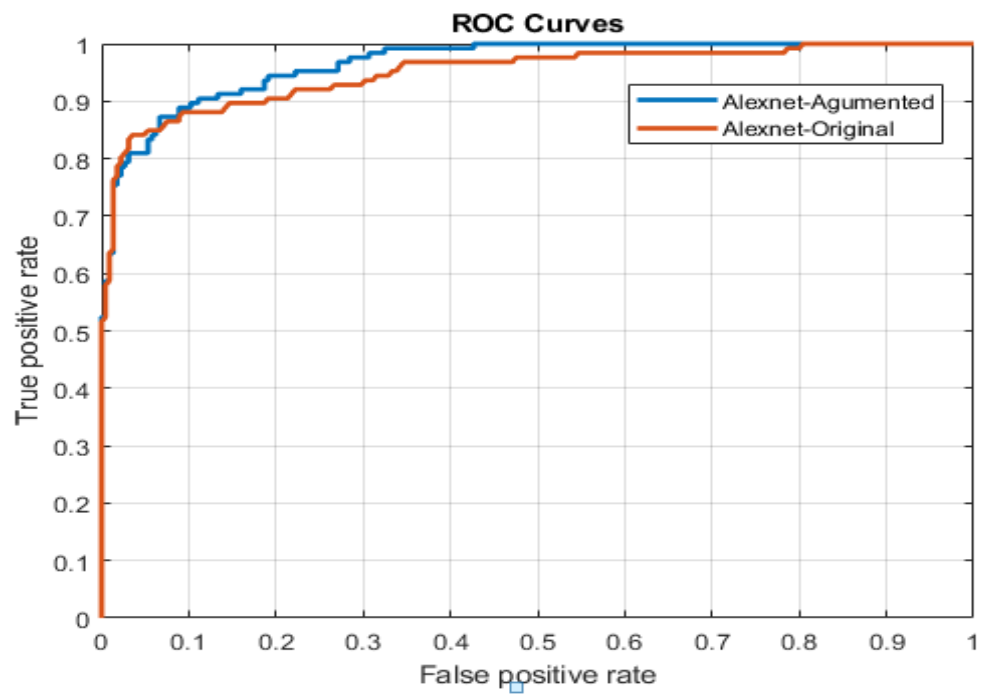

Figure 13. ROC performance for AlexNet with skip connection on original and augmented image data

We also analyze the area under the region of convergence on both original and augmented image data. The Receiving Operating Characteristics (ROC) is the graphical plot used to show the diagnostic ability of the binary classifiers. For the AlexNet, on the original data, the ROC value is 95.6 and on the augmented image data, the ROC value is obtained as 97.6. It shows that the augmented image data makes the AlexNet effective, having learned more features for better classification, as shown in Figure 13. 


\subsection{Performance of VGG16 with Skip Connection}

We have analyzed the effect of each layer in VGG16 architecture. The accuracy difference between the first and last convolutional layers is $32.5 \%$, which reveals that the first convolutional layers learning depends on the visible variations in the image, while the final convolutional layers learning depends on the image visible and invisible variations, very deeply as it gains higher accuracy.

Table 6. Performance of VGG16 with skip connection

$\begin{array}{lccccc}\text { Layers } & \begin{array}{l}\text { Accuracy } \\ (\%)\end{array} & \text { Sensitivity }(\%) & \text { Specificity }(\%) & \text { F-score }(\%) & \text { Precision (\%) } \\ \text { Conv_1 } & 50 & 55 & 78 & 59 & 56 \\ \text { Conv_3 } & 63.5 & 70.2 & 84 & 72.5 & 72 \\ \text { Conv_5 } & 65.3 & 73.2 & 85 & 73.5 & 74.5 \\ \text { Conv_7 } & 68.5 & 75 & 88 & 76.7 & 77.2 \\ \text { Conv_9 } & 72.5 & 78.5 & 88.4 & 79.2 & 81.2 \\ \text { Conv_11 } & 77.3 & 82 & 91.5 & 82.2 & 84 \\ \text { Conv_13 } & 82.5 & 88.2 & 94 & 89.5 & 91 \\ \text { FC_1 } & 96.5 & 96.2 & 95.2 & 92.5 & 92.5 \\ \text { FC_2 } & 98.9 & 96.9 & 97 & 94.2 & 94.8\end{array}$

From Table 6, after freezing some of the layers in the VGG16 architecture, the performances of convolutional layers from conv_1 to conv_13, as well as fully connected layers are examined. Each convolutional layer is learning different kind of features from the brain image. The increment of the number of convolutional layers increases the classification performance. Therefore, the final convolutional layer, i.e., conv_13 provides a good performance compared to all other convolutional layers. The final fully connected layers have higher performance compared to all other layers in the VGG16 architecture, which has an accuracy of $97.2 \%$. 

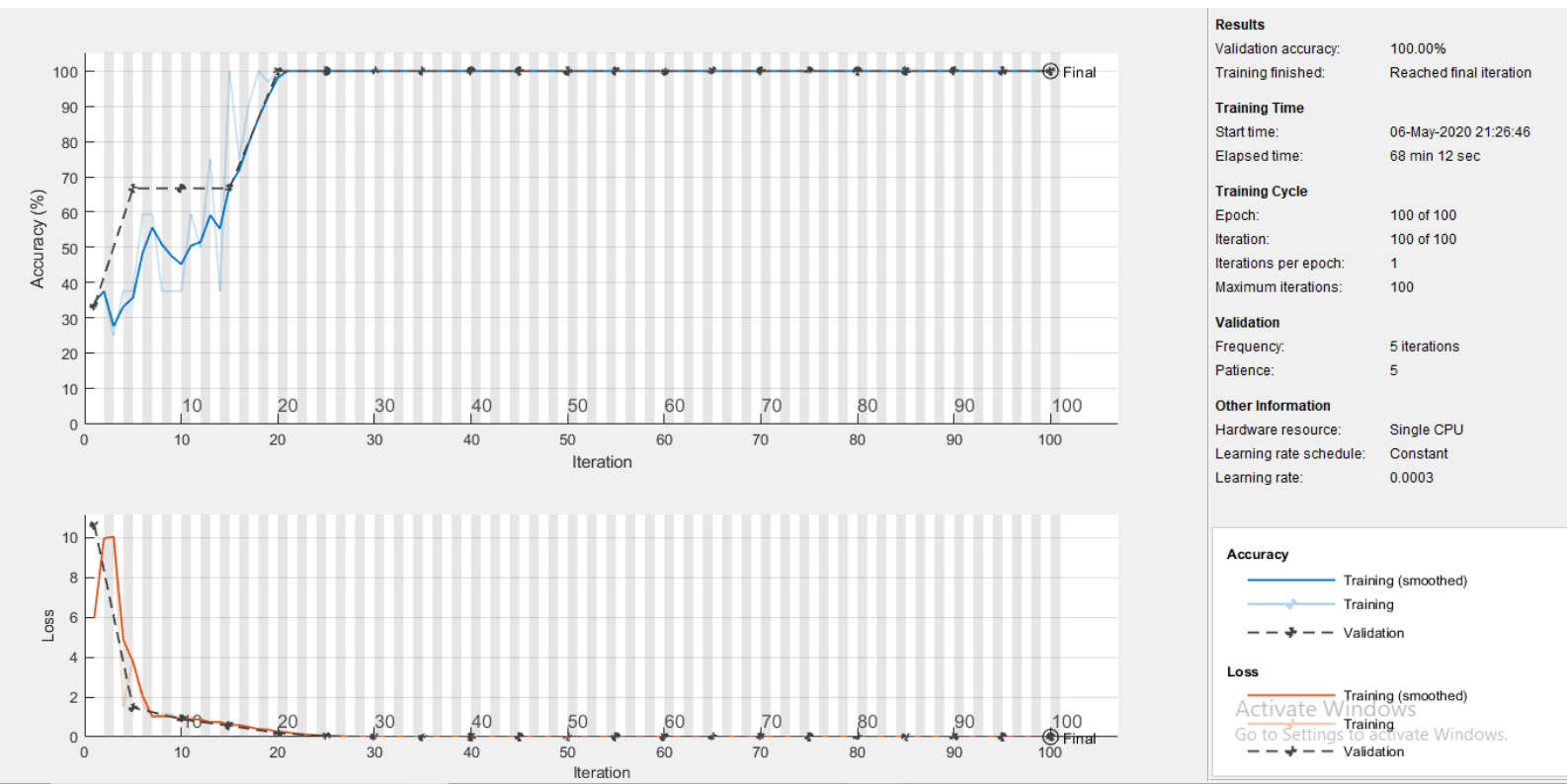

Figure 14. Training progress for VGG16 with Skip Connection

Figure 14 shows the training progress in which the training accuracy is getting $100 \%$ when the number of epochs is 200 and the loss is reducing up to 0.003. Validation accuracy is also progressing in a promising manner but it is better than AlexNet network performance.

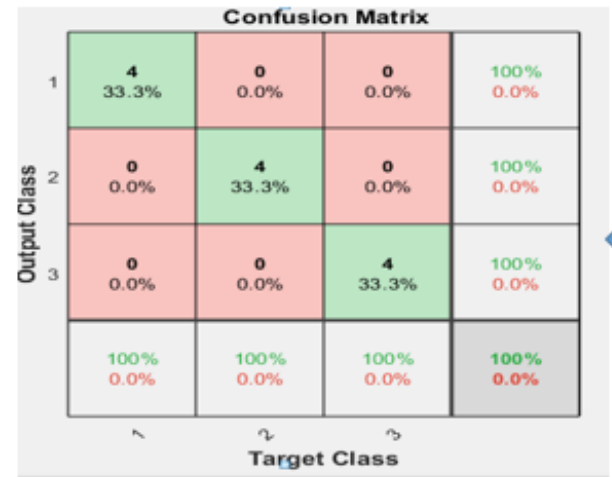

\begin{tabular}{|c|c|c|c|c|c|}
\hline & & \multicolumn{3}{|c|}{ Target Class } & \\
\hline & & Glioma & Meningioma & Pituitary & \\
\hline \multirow{4}{*}{ Output } & Glioma & 4 & 0 & 0 & $100 \%$ \\
& & $33.3 \%$ & $0.0 \%$ & $0.0 \%$ & $0.0 \%$ \\
\cline { 2 - 6 } & Meningioma & 0 & 4 & 0 & $100 \%$ \\
\cline { 3 - 6 } & & $0.0 \%$ & $33.3 \%$ & $0.0 \%$ & $0.0 \%$ \\
\cline { 2 - 6 } & Plass & 0 & 0 & 4 & $100 \%$ \\
& & $0.0 \%$ & $0.0 \%$ & $33.3 \%$ & $100 \%$ \\
& & & $5.6 \%$ & & $0.0 \%$ \\
\hline & & $100 \%$ & $100 \%$ & $100 \%$ & $100 \%$ \\
& & $0.0 \%$ & $0.0 \%$ & $0.0 \%$ & $0.0 \%$ \\
\hline
\end{tabular}

Figure 15. Confusion matrix for VGG16 with Skip Connection

The numerical computation in the confusion matrix are obtained as follows:

True Positive $(\mathrm{Tp})=4$; True Negative $(\mathrm{Tn})=4+0+0+4=8, \quad$ False Positive $(\mathrm{Fp})=0$ and False 
Negative $(\mathrm{Fn})=0$.

The True Positive $=\mathrm{Tp} /$ sample total; $4 / 12=33.33 \%$. In our case the number of trained samples has been 18 Glioma out of 20 and 17 out of Meningioma; 4/12 = 33.3.8\% and from Pituitary; $4 / 12=33.3 \%$.

From Figure 15, the digits 1, 2 and 3 represent the different classes of brain tumors, namely Glioma, Meningioma and Pituitary. In the experiment, among the 60 test samples, 6 Glioma test samples are identified as Glioma, 3 samples are identified as Meningioma, and 4 as Pituitary. Likewise, for Meningioma classes: 5 samples are accurately identified as Meningioma, 2 samples as Glioma, and 2 as Pituitary. For Pituitary class: 6 samples are identified as Pituitary, 2 samples as Glioma, and 3 samples are identified as Meningioma, respectively. Therefore, the overall accuracy for the fine-tuned VGG network is $100.00 \%$.

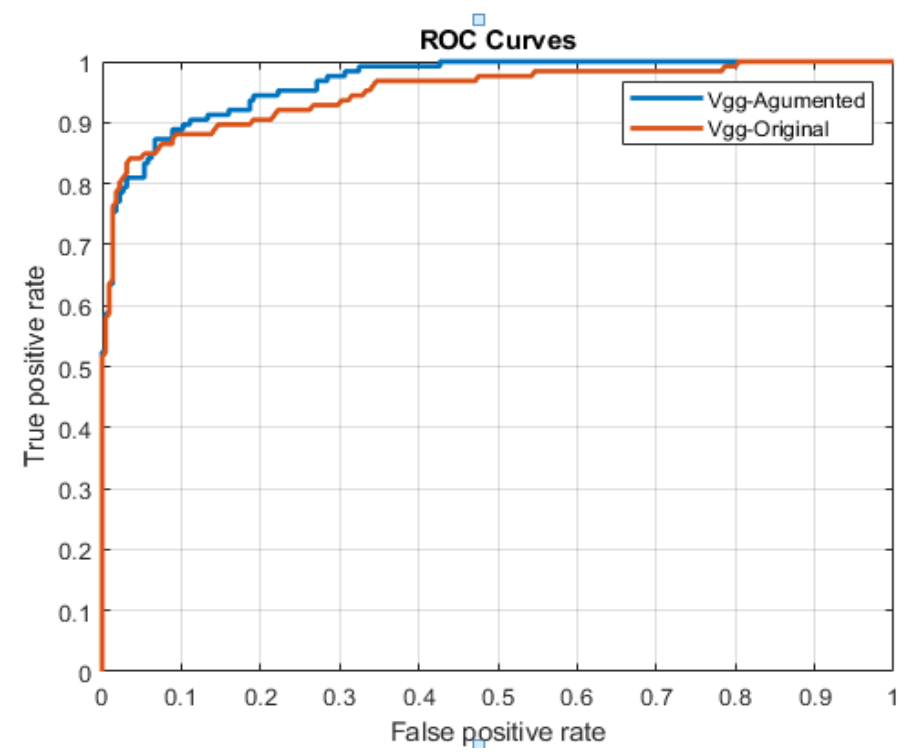

Figure 16. ROC for VGG16 with Skip Connection on original and augmented image data

For VGG16 architecture, from Figure 16, on the original data, the ROC value is 96.9, and on the augmented image data, the ROC value is obtained as 98.1. It shows that the augmented image data makes the VGG16 architcture effective, having learned more features for better classification, which is slightly better than AlexNet and lower than GoogLeNet. 


\subsection{Performance of GoogLeNet with Skip Connection}

Table 7. Performance of GoogLeNet with skip connection

$\begin{array}{lccccc}\text { Layers } & \text { Accuracy (\%) } & \text { Sensitivity (\%) } & \text { Specificity (\%) } & \text { F-score (\%) } & \text { Precision (\%) } \\ \text { Inception 3a } & 80 & 85 & 88 & 89 & 86 \\ \text { Inception 3b } & 91.5 & 90.2 & 94 & 92.5 & 92 \\ \text { Inception 4a } & 89.8 & 90 & 93.4 & 90 & 90 \\ \text { Inception 4b } & 91.1 & 90.2 & 95.2 & 91.5 & 90.7 \\ \text { Inception 4c } & 92 & 91 & 96 & 92.2 & 91 \\ \text { Inception 4d } & 94 & 92.5 & 94.1 & 94.2 & 94 \\ \text { Inception 4e } & 95.5 & 94 & 95 & 95.5 & 94.5 \\ \text { Inception 5a } & 97 & 96.2 & 96.7 & 97.1 & 96 \\ \text { Inception 5b } & 98.7 & 97 & 97.8 & 98.2 & 97.7\end{array}$

For the GoogLeNet, the performance of the different inception network layers is analyzed. From Table 7, the performances of layers such as Inception 3, Inception 4, Inception 5 sub-layers are examined. When the inception modules are increased, the features learning increases and it leads to improvement in accuracy. The last inception module $5 \mathrm{~b}$ produces better performance compared to other inception modules. Therefore, the learning of features in the last inception module is better than others. The accuracy of final inception module is 98.7 , which is better than AlexNet and VGG16 architectures.

From Table 7 we can see that the learning variations between the inception blocks are different, in which the first inception block gives accuracy of $80 \%$ and the performance increases for consecutive inception module and the final modules gives accuracy of $97 \%$. The difference is $16 \%$, which is less compared to VGG16 and AlexNet architectures. 

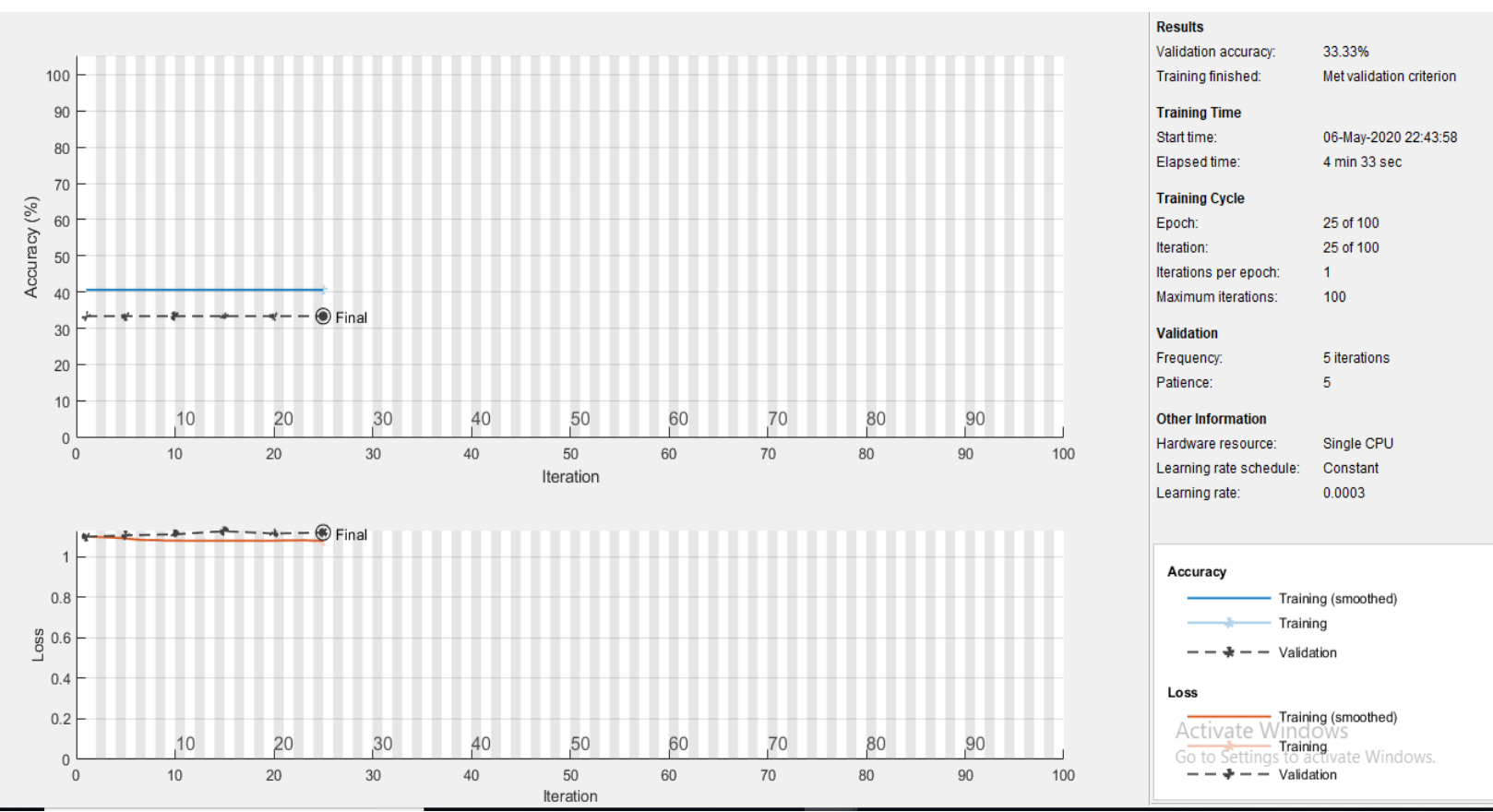

Figure-17. Training progress for GoogLeNet with Skip Connection

GoogLeNet training progress and validation performance are higher than both AlexNet and VGG16 architectures. Figure 17 shows loss is reducing up to 0.0005 . Here, the learning rate is 0.0003 for final epoch 100 .

\begin{tabular}{|c|c|c|c|c|}
\hline \multirow{6}{*}{ 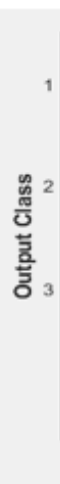 } & \multicolumn{4}{|c|}{ Confusion Matrix } \\
\hline & $\begin{array}{c}4 \\
33.3 \%\end{array}$ & 33. 4 & $\begin{array}{c}4 \\
33.3 \%\end{array}$ & $\begin{array}{l}33.3 \% \\
66.7 \%\end{array}$ \\
\hline & $\begin{array}{c}0 \\
0.0 \%\end{array}$ & $\begin{array}{c}0 \\
0.0 \%\end{array}$ & $\begin{array}{c}0 \\
0.0 \%\end{array}$ & $\begin{array}{l}\text { NaN\% } \\
\text { NaN\% }\end{array}$ \\
\hline & $\begin{array}{c}0 \\
0.0 \%\end{array}$ & $\begin{array}{c}0 \\
0.0 \%\end{array}$ & $\begin{array}{c}0 \\
0.0 \%\end{array}$ & $\begin{array}{l}\text { NaN\% } \\
\text { NaN\% }\end{array}$ \\
\hline & $\begin{array}{l}100 \% \\
0.0 \%\end{array}$ & $\begin{array}{l}0.0 \% \\
100 \%\end{array}$ & $\begin{array}{l}0.0 \% \\
100 \%\end{array}$ & $\begin{array}{l}33.3 \% \\
66.7 \%\end{array}$ \\
\hline & \multicolumn{4}{|c|}{${ }_{\text {Target Class }}^{2}$} \\
\hline
\end{tabular}

\begin{tabular}{|c|c|c|c|c|c|}
\hline & & \multicolumn{3}{|c|}{ Target Class } & \\
\hline & & Glioma & Meningioma & Pituitary & \\
\hline \multirow{3}{*}{$\begin{array}{l}\text { Output } \\
\text { Class }\end{array}$} & Glioma & $\begin{array}{c}4 \\
33.3 \% \\
\end{array}$ & $\begin{array}{c}4 \\
33.3 \% \\
\end{array}$ & $\begin{array}{c}4 \\
33.3 \% \\
\end{array}$ & $\begin{array}{l}33.3 \% \\
67.7 \% \\
\end{array}$ \\
\hline & Meningioma & $\begin{array}{c}0 \\
0.0 \%\end{array}$ & $\begin{array}{c}4 \\
33.3 \% \\
\end{array}$ & $\begin{array}{c}0 \\
0.0 \%\end{array}$ & $\begin{array}{l}\mathrm{NaN} \\
\mathrm{NaN}\end{array}$ \\
\hline & Pituitary & $\begin{array}{c}0 \\
0.0 \%\end{array}$ & $\begin{array}{c}0 \\
0.0 \% \\
5.6 \%\end{array}$ & $\begin{array}{c}4 \\
33.3 \%\end{array}$ & $\begin{array}{l}\mathrm{NaN} \\
\mathrm{NaN}\end{array}$ \\
\hline & & $\begin{array}{l}0.0 \% \\
100 \%\end{array}$ & $\begin{array}{l}0.0 \% \\
100 \%\end{array}$ & $\begin{array}{l}0.0 \% \\
100 \%\end{array}$ & $\begin{array}{l}33.3 \% \\
67.7 \%\end{array}$ \\
\hline
\end{tabular}

Figure 18. Confusion matrix for GoogLeNet with skip connection 
Figure 18 shows the confusion matrix for GoogLeNet with skip connection. In the experiment, among the 60 test samples, 6 Glioma test samples are identified as Glioma, 3 samples are identified as Meningioma, and 4 as Pituitary. Likewise, for Meningioma classes, 5 samples are accurately identified as Meningioma, 2 samples as Glioma, and 2 as Pituitary. For Pituitary class, 6 samples are identified as Pituitary, 2 samples as Glioma, and 3 samples are identified as Meningioma, respectively. So, the overall accuracy for the fine-tuned GoogLeNet is 98.5\%. In Fig. 18, NaN means not a number.

For GoogLeNet, from Figure 19, on the original data, the ROC value is 97.9 and on the augmented image data, the ROC value is obtained as 98.6, which is better compared to the other two networks, AlexNet and VGG16 architectures.

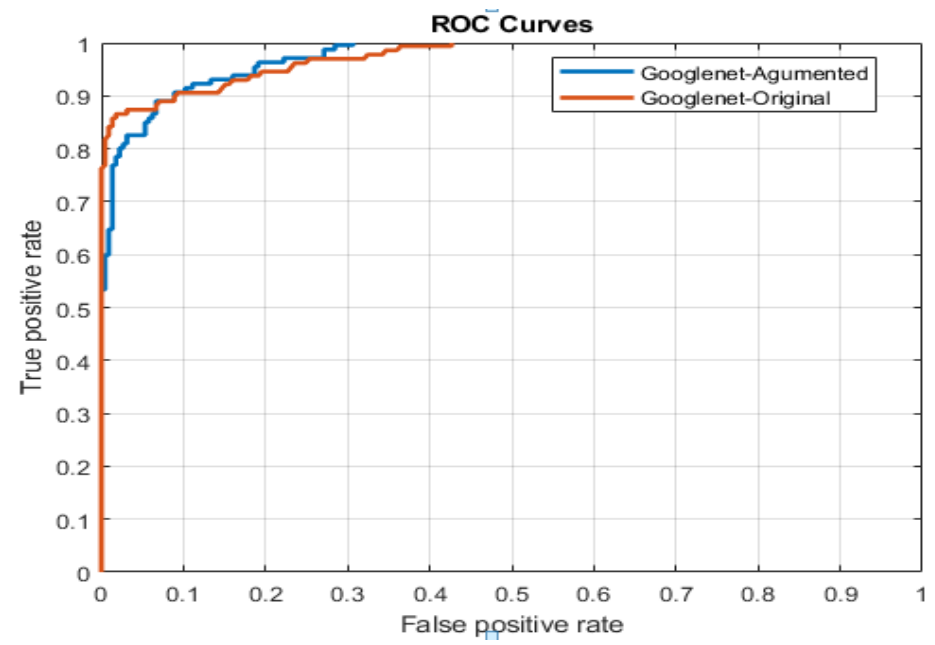

Figure 19. ROC performance for GoogLeNet with skip connection on original and augmented image data

\section{Discussion with Related Work}

When compared with the existing methods [34-36], the proposed skip-forward models perform well in terms of all the performance metrics. In the previous work, they have got the accuracy for all the three CNN architectures, AlexNet, VGG, GoogLeNet as 97.39, 98.69, and 98.04, respectively. But in our case, we got 97.60, 98.99, 98.50, respectively, because we have implemented the frequency domain information enhancement in the pre-processing and changing 
the potential parameters to fine-tune the network, which helps in improving the classification performance

In references [34-36], the three CNN architectures were fine-tuned all by changing the final fully connected layer of the network to 3 for three different classes. Also, the experiments were carried out by freezing the layers for different networks. Consequently, there is a chance of getting vanishing moments and overfitting the feature maps.

However, in this paper, a transfer learning model is presented via CNN architectures with skip or shot connections based on the features learning capabilities of all the three pre-trained networks. We have analyzed the layer by layer outcome and the feature map for all intermediate layers, and introduced the shot connections where ever needed. This leads to better performance. Also, we have employed a robust frequency domain information enhancement technique to make image information more visible for feature extraction in the pre-processing. These two modifications have led to good results in the system performance.

Table 8 shows the performance metrics of the three CNN architectures with skip connections employed in this paper, in comparison to the existing models in references [34-36], while Figure 20 shows the performances of all the transfer learning models.

Table 8. Comparison of the transfer learning models

\begin{tabular}{|c|c|c|c|c|}
\hline $\begin{array}{c}\text { Accuracy } \% \\
\backslash \\
\text { Models }\end{array}$ & & AlexNet & VGG16 & GoogLeNet \\
\hline [35] & \multirow{3}{*}{$\begin{array}{l}\text { No skip } \\
\text { connection }\end{array}$} & 93.33 & 96.25 & 97.1 \\
\hline [36] & & 97.5 & 95 & 95 \\
\hline [34] & & 97.39 & 98.69 & 98.04 \\
\hline our TL model & $\begin{array}{l}\text { CNN with skip } \\
\text { connection }\end{array}$ & 97.6 & 98.92 & 98.3 \\
\hline
\end{tabular}


From Table 8 and Figure 20, the VGG16 with shot connection performs well, that is accuracy of $98.92 \%$ compared to the other two architectures, AlexNet and GoogLeNet with shot connection. Also, when compared to the existing models [34-36], the VGG16 with shot connection gives a 0.33 percentage improvement in terms of accuracy.

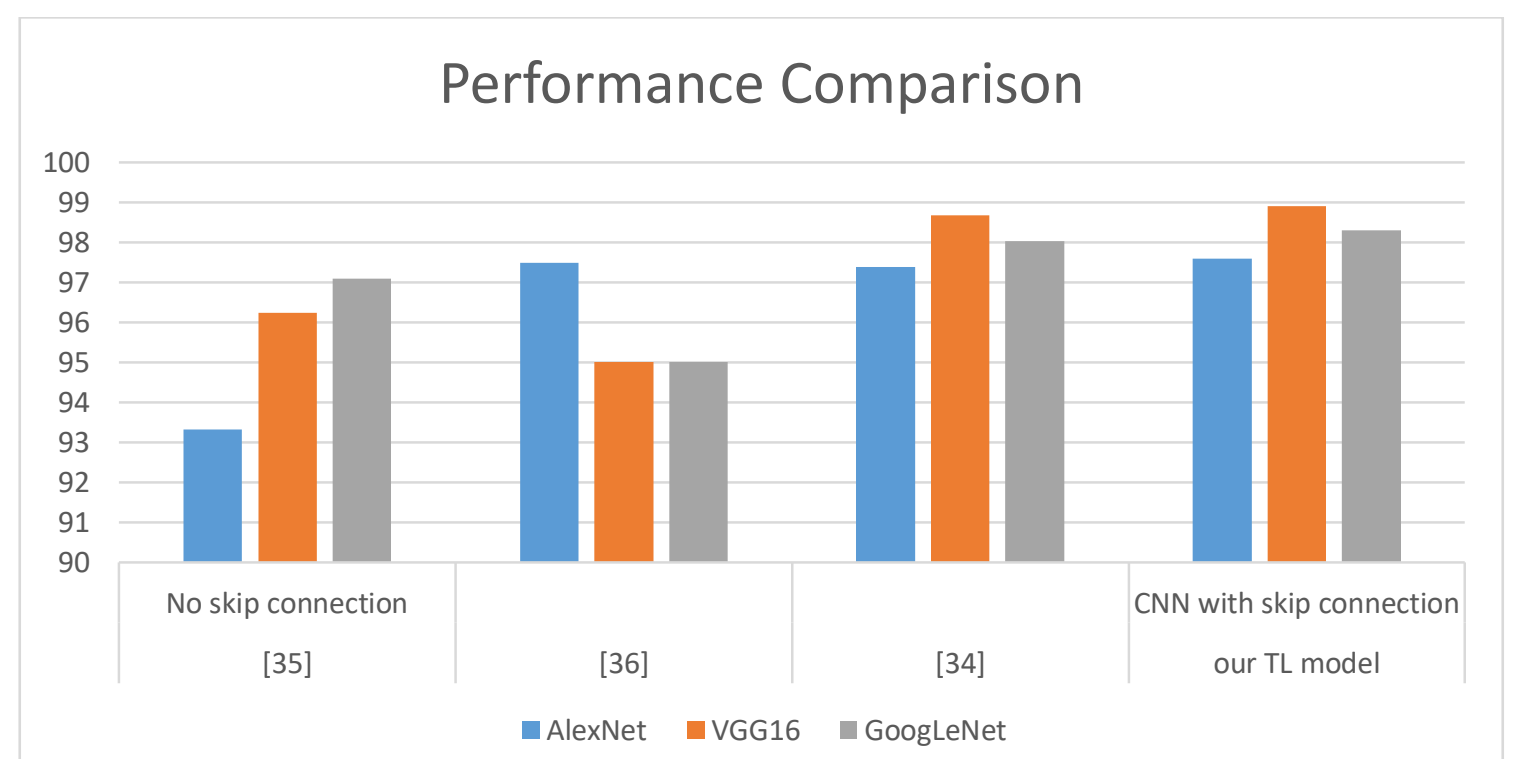

Figure 20. Comparison of transfer learning models

Our transfer learning model via CNN with skip connection has the advantages of avoiding the vanishing gradients and time complexity commonly with transfer learning networks. In addition, our transfer learning model via $\mathrm{CNN}$ with skip connection has led to the better classifications of brain tumor, with higher accuracy than the existing models. Moreover, the use of frequency domain information enhancement technique in the pre-processing has led to better image clarity and has helped improve performance of brain tumor classifications. On the other hand, the major shortcoming of our transfer learning models via CNN with skip connection is that only one dataset is used in the experimental validations with limited input classes.

\section{Conclusion}

This paper has presented a Transfer Learning (TL) model via CNN with skip connection to detect the abnormality in the brain Magnetic Resonance Imaging (MRI). This paper 
employs three CNN architectures, i.e., AlexNet, VGG16 and GoogLeNet, to implement the transfer learning model based on the MRI slices of brain tumor dataset for brain tumor classifications. The CNN architectures with skip connections are able to:

- Attain highest accuracy of 98.92 for the VGG16 with skip connection in brain tumor classification, , and

- Reduce the vanishing gradient and time complexity commonly with transfer learning networks,

When implementing transfer leaning on based on the MRI slices of brain tumor dataset, it is good for $n$ the performance of brain tumor classifications to employ the frequency domain information enhancement technique in the pre-processing as it give better images clarity.

For future work, implementing the transfer learning model may involve:

- use of the ensemble classifiers to improve the classification accuracy, and

- extension to a multi-class classifier for fine-tuing and freezing.

The transfer learning model can be implemented in other applications such as COVID-19 MRI images, skin cancer and other types of medical image applications.

\section{Statement of Contribution}

The following are the contributions of the authors to work:

Saleh Alaraimi: conceptualization of the work and simulations analysis.

Kenneth E. Okedu: literature review, data analysis and writing of the paper

Richard Holden: data analysis of the work

Omair Uthmani: literature review of the work

Hugo Tianfield: supervision, data analysis and writing of the work 


\section{References}

Alafaci, C., Granata, F., Cutugno, M., Caffo, M., Caruso, G. and Salpietro, F.M., 2013. Modern Neuroimaging Techniques in the Diagnosis of Brain Tumor. Clinical Management and Evolving Novel Therapeutic Strategies for Patients with Brain Tumors, p.55.

[2]. Alqudah, A.M., Alquraan, H., Qasmieh, I.A., Alqudah, A. and Al-Sharu, W., 2020. Brain Tumor Classification Using Deep Learning Technique--A Comparison between Cropped, Uncropped, and Segmented Lesion Images with Different Sizes. arXiv preprint arXiv:2001.08844.

[3]. Ari, A. and Hanbay, D., 2018. Deep learning-based brain tumor classification and detection system. Turkish Journal of Electrical Engineering \& Computer Sciences, 26(5), pp.2275-2286.

[4]. Azer, S.A., 2019. Deep learning with convolutional neural networks for identification of liver masses and hepatocellular carcinoma: A systematic review. World Journal of Gastrointestinal Oncology, 11(12), p.1218.

[5]. Banerjee, S., Mitra, S., Sharma, A. and Shankar, B.U., 2018. A CADe system for gliomas in brain MRI using convolutional neural networks. arXiv preprint arXiv:1806.07589.

[6]. Carass, A., Wheeler, M.B., Cuzzocreo, J., Bazin, P.L., Bassett, S.S. and Prince, J.L., 2007, April. A joint registration and segmentation approach to skull stripping. In 2007 4th IEEE International Symposium on Biomedical Imaging: From Nano to Macro (pp. 656-659). IEEE.

[7]. Chaddad, A. and Tanougast, C., 2016. Quantitative evaluation of robust skull stripping and tumor detection applied to axial MR images. Brain informatics, 3(1), pp.53-61.

[8]. Chato, L. and Latifi, S., 2017, October. Machine learning and deep learning techniques to predict overall survival of brain tumor patients using MRI images. In 2017 IEEE 17th International Conference on Bioinformatics and Bioengineering (BIBE) (pp. 9-14). IEEE. 
[9]. Chollet, F., 2017. Xception: Deep learning with depthwise separable convolutions. In Proceedings of the IEEE conference on computer vision and pattern recognition (pp. 1251-1258).

[10]. Dallora, A.L., Eivazzadeh, S., Mendes, E., Berglund, J. and Anderberg, P., 2017. Machine learning and microsimulation techniques on the prognosis of dementia: A systematic literature review. PloS one, 12(6).

[11]. Despotović, I., Goossens, B. and Philips, W., 2015. MRI segmentation of the human brain: challenges, methods, and applications. Computational and mathematical methods in medicine, 2015.

[12]. Dolgushin, M., Kornienko, V. and Pronin, I., 2018. Introduction to Neuroimaging Techniques in the Diagnosis of Brain Cancer Metastases. In Brain Metastases (pp. 27-27). Springer, Cham.

[13]. Durmo, F., Lätt, J., Rydelius, A., Engelholm, S., Kinhult, S., Askaner, K., Englund, E., Bengzon, J., Nilsson, M., Björkman-Burtscher, I.M. and Chenevert, T., 2018. Brain tumor characterization using multibiometric evaluation of MRI. Tomography, 4(1), p.14.

[14]. El Kader Isselmou, A., Xu, G., Zhang, S., Saminu, S. and Javaid, I., 2019, July. Deep Learning Algorithm for Brain Tumor Detection and Analysis Using MR Brain Images. In Proceedings of the 2019 International Conference on Intelligent Medicine and Health (pp. 28-32).

[15]. Farmanfarma, K.K., Mohammadian, M., Shahabinia, Z., Hassanipour, S. and Salehiniya, H., 2019. Brain Cancer In The World: An Epidemiological Review. World Cancer Research Journal, 6, p.5.

[16]. Fischmeister, F.P.S., Höllinger, I., Klinger, N., Geissler, A., Wurnig, M.C., Matt, E., Rath, J., Robinson, S.D., Trattnig, S. and Beisteiner, R., 2013. The benefits of skull stripping in the normalization of clinical fMRI data. NeuroImage: Clinical, 3, pp.369380. 
[17]. Gilanie, G., Bajwa, U.I., Waraich, M.M., Habib, Z., Ullah, H. and Nasir, M., 2018. Classification of normal and abnormal brain MRI slices using Gabor texture and support vector machines. Signal, Image and Video Processing, 12(3), pp.479-487.

[18]. Kuklisova-Murgasova, M., Quaghebeur, G., Rutherford, M.A., Hajnal, J.V. and Schnabel, J.A., 2012. Reconstruction of fetal brain MRI with intensity matching and complete outlier removal. Medical image analysis, 16(8), pp.1550-1564.

[19]. Lin, W., Tong, T., Gao, Q., Guo, D., Du, X., Yang, Y., Guo, G., Xiao, M., Du, M., Qu, X. and Alzheimer's Disease Neuroimaging Initiative, 2018. Convolutional neural networks-based MRI image analysis for the Alzheimer's disease prediction from mild cognitive impairment. Frontiers in neuroscience, 12, p.777.

[20]. Litjens, G., Sánchez, C.I., Timofeeva, N., Hermsen, M., Nagtegaal, I., Kovacs, I., Hulsbergen-Van De Kaa, C., Bult, P., Van Ginneken, B. and Van Der Laak, J., 2016. Deep learning as a tool for increased accuracy and efficiency of histopathological diagnosis. Scientific reports, 6, p.26286.

[21]. Madhupriya, G., Guru, N.M., Praveen, S. and Nivetha, B., 2019, April. Brain Tumor Segmentation with Deep Learning Technique. In 2019 3rd International Conference on Trends in Electronics and Informatics (ICOEI) (pp. 758-763). IEEE.

[22]. Mohsen, H., El-Dahshan, E.S.A., El-Horbaty, E.S.M. and Salem, A.B.M., 2018. Classification using deep learning neural networks for brain tumors. Future Computing and Informatics Journal, 3(1), pp.68-71.

[23]. Munir, K., Elahi, H., Ayub, A., Frezza, F. and Rizzi, A., 2019. Cancer Diagnosis Using Deep Learning: A Bibliographic Review. Cancers, 11(9), p.1235.

[24]. Park, B.Y., Byeon, K. and Park, H., 2019. FuNP (fusion of neuroimaging preprocessing) pipelines: a fully automated preprocessing software for functional magnetic resonance imaging. Frontiers in neuroinformatics, 13, p.5. 
[25]. Pugalenthi, R., Rajakumar, M.P., Ramya, J. and Rajinikanth, V., 2019.

Evaluation and Classification of the Brain Tumor MRI using Machine Learning Technique. Journal of Control Engineering and Applied Informatics, 21(4), pp.12-21.

[26]. Rajasekaran, K.A. and Gounder, C.C., 2018. Advanced

Brain Tumor Segmentation from MRI Images. High-Resolution Neuroimaging: Basic Physical Principles and Clinical Applications, p.83.

[27]. Roy, S. and Maji, P., 2015, January. A simple skull stripping algorithm for brain MRI. In 2015 Eighth International Conference on Advances in Pattern Recognition (ICAPR) (pp. 1-6). IEEE.

[28]. Sobhaninia, Z., Rezaei, S., Noroozi, A., Ahmadi, M., Zarrabi, H., Karimi, N., Emami, A. and Samavi, S., 2018. Brain tumor segmentation using deep learning by type specific sorting of images. arXiv preprint arXiv:1809.07786.

[29]. Suk, H.I., Shen, D. and Alzheimer's disease Neuroimaging Initiative, 2015.

Deep learning in diagnosis of brain disorders. In Recent Progress in Brain and Cognitive Engineering (pp. 203-213). Springer, Dordrecht.

[30]. Swiebocka-Wiek, J., 2016, September. Skull stripping for MRI images using morphological operators. In IFIP International Conference on Computer Information Systems and Industrial Management (pp. 172-182). Springer, Cham.

[31]. Tandel, G.S., Biswas, M., Kakde, O.G., Tiwari, A., Suri, H.S., Turk, M., Laird, J.R., Asare, C.K., Ankrah, A.A., Khanna, N.N. and Madhusudhan, B.K., 2019. A review on a deep learning perspective in brain cancer classification. Cancers, 11(1), p.111.

[32]. Widhiarso, W., Yohannes, Y. and Prakarsah, C., 2018. Brain tumor classification using gray level co-occurrence matrix and convolutional neural network. IJEIS (Indones. J. Electron. Instrum. Syst.), 8(2), pp.179-190.

[33]. Szegedy, C., et al. 2015: Going deeper with convolutions. In: Proceedings of the IEEE Conference on Computer Vision and Pattern Recognition. 
[34]. Rehman, A. et al. (2020) 'A Deep Learning-Based Framework for Automatic Brain Tumors Classification Using Transfer Learning', Circuits, Systems, and Signal Processing. Springer US, 39(2), pp. 757-775. doi: 10.1007/s00034-019-01246-3.

[35]. Bhanumathi, V. and Sangeetha, R. (2019) 'CNN Based Training and Classification of MRI Brain Images’, 2019 5th International Conference on Advanced Computing and Communication Systems, ICACCS 2019. IEEE, pp. 129-133. doi: 10.1109/ICACCS.2019.8728447.

[36]. Revi K, R. and Wilscy, M. (2018) 'Pretrained Convolutional Neural Networks as Feature Extractor for Image Splicing Detection', 2018 International Conference on Circuits and Systems in Digital Enterprise Technology, ICCSDET 2018. IEEE, pp. 04. doi: 10.1109/ICCSDET.2018.8821242.

[37]. He, K. et al. (2016) 'Deep Residual Learning for Image Recognition', Proceeding of the IEEE Computer Society Conference on Computer vision and Patterns Recognition, 2016-December, pp. 770-778. Doi: 10-1109/CVRP. 2016.90. [38]. Cheng J. (2018)

'Brain tumor dataset. figshare.data. https://doi.org/10.6084/m9.figshare.1512427.v5. Accessed May 30, 2018.

[39]. Szegedy, C. et al. (2015) 'Going deeper with convolutions', Proceedings of the IEEE Computer Society Conference on Computer Vision and Pattern Recognition, 0712-June-2015, pp. 1-9. doi: 10.1109/CVPR.2015.7298594.

[40]. Yuan, L., Wei, X., Shen, H., Zeng, L.L. and Hu, D., 2018. Multi-center brain imaging classification using a novel 3D CNN approach. IEEE Access, 6, pp.4992549934.

[41]. Gonzalez, T. F. (2007) 'Handbook of approximation algorithms and metaheuristics', Handbook of Approximation Algorithms and Metaheuristics, pp. 11432. doi: $10.1201 / 9781420010749$. 
[42]. Yang, Y., Yan, L.F., Zhang, X., Han, Y., Nan, H.Y., Hu, Y.C., Hu, B., Yan, S.L., Zhang, J., Cheng, D.L. and Ge, X.W., 2018. Glioma grading on conventional MR images: a deep learning study with transfer learning. Frontiers in neuroscience, 12, p.804.

[43]. Simonyan, K. and Zisserman, A. (2015) 'Very deep convolutional networks for large-scale image recognition', 3rd International Conference on Learning Representations, ICLR 2015 - Conference Track Proceedings, pp. 1-14. 